\title{
Trajectory Sensitivity Analysis of Hybrid Systems
}

\author{
Ian A. Hiskens, Senior Member, IEEE, and M. A. Pai, Fellow, IEEE
}

\begin{abstract}
The development of trajectory sensitivity analysis for hybrid systems, such as power systems, is presented in the paper. A hybrid system model which has a differential-algebraic-discrete (DAD) structure is proposed. This model forms the basis for the subsequent sensitivity analysis. Crucial to the analysis is the development of jump conditions describing the behavior of sensitivities at discrete events, such as switching and state resetting. The efficient computation of sensitivities is discussed. A number of examples are presented to illustrate various aspects of the theory. It is shown that trajectory sensitivities provide insights into system behavior which cannot be obtained from traditional simulation.
\end{abstract}

Index Terms-Dynamic response, hybrid dynamical systems, power systems, switched systems, trajectory sensitivity.

\section{INTRODUCTION}

$\mathbf{M}$ ANY PHYSICAL systems exhibit dynamic behavior which is governed by a mix of continuous-time (possibly constrained) dynamics, discrete-time and discrete-event dynamics, switching action, and jump phenomena. Such systems range from batch processes [1] through to power systems [2] and have become known generically as hybrid systems. Some simple examples which illustrate various aspects of hybrid systems are given in Sections II and VI.

Consider power systems, for example. The behavior of such systems is governed by the nonlinear dynamics of many components, including machines, loads, flexible AC transmission system (FACTS) devices, and their associated control equipment. Dynamic behavior is constrained by physical laws; for example, current balance must be maintained at all nodes. Furthermore, protection relays, controller limits, and discrete devices, such as on-load tap changing transformers and switched shunts, introduce discrete events, switching action, and state resetting into the system. Power system behavior can therefore be quite complicated, yet system integrity is reliant on a thorough understanding of that behavior. This requires effective and insightful analysis.

The nonlinear nonsmooth dynamics of hybrid systems make analysis difficult. While Lyapunov-type theory offers analytical insights for specific applications (see, for example, [1], [3], and [4]), in general, the analysis of hybrid systems is reliant on time-domain simulation. The advantage of simulation is that it is applicable for arbitrarily complicated models. A disadvantage is that it provides information about a single scenario. Generally it is not possible to confidently extrapolate results, even for

Manuscript received January 20, 1998; revised October 1998 and April 7, 1999. This work was supported in part by the Australian Research Council under Project Grant Analysis and Assessment of Voltage Collapse and in part by the National Science Foundation under Grant NSF ECS 98-03055 and the Grainger Foundation. This paper was recommended by Associate Editor J. Suykens.

The authors are with the Department of Electrical and Computer Engineering, University of Illinois at Urbana-Champaign, Urbana, IL 61801 USA.

Publisher Item Identifier S 1057-7122(00)01500-2. small changes in system conditions. Each change to the system requires another simulation. For large systems, such as power systems, this often involves large computational costs.

Trajectory sensitivity analysis offers some relief from the rigors of repetitive simulation. The approach is based upon linearizing the system around a nominal trajectory rather than around an equilibrium point. It is therefore possible to determine directly the change in a trajectory due to (small) changes in initial conditions and/or parameters. These concepts are well established for continuous dynamics [5]-[8], while closely related perturbation analysis theory has been developed for discrete event dynamic systems (DEDS's) [10], [11]. An interesting comparison of these fields is presented in [12]. This paper makes an important extension to a general hybrid system model.

Trajectory sensitivities provide valuable insights into the influence of parameters on the dynamic behavior of systems. Properties which are not obvious from the actual system response are often evident in the sensitivities. For example, the extra information available from trajectory sensitivities was used in [13] to investigate the relative importance of various events in a major power system disturbance. The example of Section VI-C further illustrates this use of trajectory sensitivities.

Trajectory sensitivities were originally associated with a number of areas in control and parameter estimation [5], [14]. More recent applications have included stability assessment of power systems [15], [16]. The ideas presented in this paper form a basis for the extension of these applications to hybrid systems.

The paper is organized as follows: Section II presents a general hybrid system model, along with a number of diverse examples. Trajectory sensitivities are introduced in Section III and their calculation away from discrete events is discussed in Section IV. The extension to discrete events, and hence general hybrid systems, is presented in Section V. Some examples are given in Section VI. Section VII discusses numerical integration issues related to the computation of sensitivities. Conclusions are drawn in Section VIII.

\section{MODEL}

\section{A. Background}

As indicated in Section I, hybrid systems are characterized by the following:

- continuous and discrete states;

- continuous dynamics;

- discrete events, or triggers;

- mappings that define the evolution of discrete states at events. 
Conceptually, such systems can be thought of as an indexed collection of continuous dynamical systems $\dot{x}=f_{q}(x)$, along with a mechanism for jumping between those systems, i.e., for switching between the various $f_{q}$. The continuous and dynamic states are $x$ and $q$, respectively. The jumping reflects the influence of the discrete event behavior and is dependent upon both the trigger condition and the discrete state evolution mapping. Overall system behavior can be viewed as a sequential patching together of dynamical systems, with the final state of one dynamical system specifying the initial state for the next.

A formal presentation of these concepts is given in [17], where a general hybrid dynamical system is defined as

$$
H=[Q, \Sigma, A, G]
$$

and

- $Q$ is the set of discrete states;

- $\Sigma=\left\{\Sigma_{q}\right\}_{q \in Q}$ is the collection of dynamical systems $\Sigma_{q}=\left[X_{q}, \Gamma_{q}, f_{q}\right]$ where each $X_{q}$ is an arbitrary topological space forming the continuous state space of $\Sigma_{q}, \Gamma_{q}$ is a semigroup over which the states evolve, and $f_{q}$ generates the continuous state dynamics;

- $A=\left\{A_{q}\right\}_{q \in Q}, A_{q} \subset X_{q}$ for each $q \in Q$, is the collection of autonomous jump sets, i.e., the conditions which trigger jumps;

- $G=\left\{G_{q}\right\}_{q \in Q}$, where $G_{q}: A_{q} \rightarrow S=\bigcup_{q \in Q}\left(X_{q} \times\{q\}\right)$ is the autonomous jump transition map.

The hybrid state-space of $H$ is given by $S$. In this paper we restrict attention to hybrid systems where $Q$ is countable, each $X_{q} \subset \mathbb{R}^{n_{q}}, n_{q} \in \mathbb{Z}_{+}$, and each $\Gamma_{q}=\mathbb{R}_{+}$.

Other representations of hybrid systems have appeared (see, for example, [18]). While each representation has its own particular flavor, they all capture the fundamental aspects of hybrid systems identified at the beginning of this section.

The level of abstraction of (1) does not suit the development of trajectory sensitivity analysis. A model which is more conducive to such analysis is presented in the following subsection. It is then shown that the proposed model is a realization of (1).

\section{B. System Description}

Many different types of systems, from manufacturing systems to power systems, can be generically described by a parameterdependent differential-algebraic-discrete (DAD) model of the form

$$
\begin{aligned}
\dot{x} & =f(x, y, z ; \lambda) \\
0 & =g^{(0)}(x, y, z ; \lambda) \\
0 & =\left\{\begin{array}{l}
g^{(i-)}(x, y, z ; \lambda) y_{d, i}<0, \quad i=1, \cdots, d \\
g^{(i+)}(x, y, z ; \lambda) y_{d, i}>0,
\end{array}\right. \\
z^{+} & =h_{j}\left(x^{-}, y^{-}, z^{-} ; \lambda\right) y_{e, j}=0, \quad j \in\{1, \cdots, e\} \\
\dot{z} & =0 \quad y_{e, j} \neq 0, \forall j \in\{1, \cdots, e\}
\end{aligned}
$$

where

$$
\begin{aligned}
& x \in X \subseteq \mathbb{R}^{n}, \quad y \in Y \subseteq \mathbb{R}^{m}, \quad z \in Z \subseteq \mathbb{R}^{l}, \quad \lambda \in L \subseteq \mathbb{R}^{p} \\
& y_{d}=D y \\
& y_{e}=E y
\end{aligned}
$$

$$
\begin{aligned}
& f: \mathbb{R}^{n+m+l+p} \rightarrow \mathbb{R}^{n} \\
& g=\left[\begin{array}{c}
g^{(0)} \\
g^{(1)} \\
\vdots \\
g^{(d)}
\end{array}\right]: \mathbb{R}^{n+m+l+p} \rightarrow \mathbb{R}^{m} \\
& h_{j}: \mathbb{R}^{n+m+l+p} \rightarrow \mathbb{R}^{l}, \quad j=1, \cdots, e
\end{aligned}
$$

and $D \in \mathbb{R}^{d \times m}, E \in \mathbb{R}^{e \times m}$ are matrices of zeros, except that each row of each matrix has a single 1 in an appropriate location. There is no restriction on $y_{d}$ and $y_{e}$ sharing some common elements. In (5), $x^{-}, y^{-}, z^{-}$refer to the values of $x, y$, and $z$ just prior to the reset condition, while $z^{+}$denotes the value of $z$ just after the reset event.

In this model, which is similar to a model proposed in [19], $x$ are continuous dynamic state variables, $y$ are algebraic state variables, $z$ are discrete state variables, and $\lambda$ are parameters. As an example, in the power system context $x$ would include machine dynamic states such as angles, velocities, and fluxes, $y$ would include network variables such as load bus voltage magnitudes and angles, $z$ could represent transformer tap positions and/or relay internal states, and $\lambda$ could be chosen from a diverse range of parameters, from loads through to fault clearing time.

Note that the model does not allow discontinuities in the dynamic states, i.e., impulse effects. This is not a restriction forced by the analysis. In fact, later analysis is directly applicable to cases where $x$ undergoes jumps. However the model adopts the philosophy that the dynamic states of real systems cannot undergo step changes.

The proposed model (2)-(6) captures all the important aspects of hybrid system behavior, namely, the interaction between continuous and discrete states as they evolve over time. Between events, system behavior is governed by the differential-algebraic (DA) dynamical system

$$
\begin{aligned}
& \dot{x}=f(x, y, z ; \lambda) \\
& 0=g_{q}(x, y, z ; \lambda)
\end{aligned}
$$

where $g_{q}$ is composed of $g^{(0)}$, together with functions from (4) chosen depending on the signs of the elements of $y_{d}$. Each different composition of $g_{q}$ is indexed by a unique $q$. An event is triggered by an element of $y_{d}$ changing sign and/or an element of $y_{e}$ passing through zero. At an event, the composition of $g_{q}$ changes and/or elements of $z$ are reset. Therefore, in this hybrid system model, each DA dynamical system is effectively indexed by $q$ and $z$. At an event, this index changes and a jump is made to the new dynamical system.

The implicit function theorem [20] allows (8) to be solved (locally) giving

$$
y=\varphi_{(q, z)}(x ; \lambda)
$$

Substitution into (7) yields

$$
\dot{x}=f_{(q, z)}^{*}(x ; \lambda) .
$$

(More complete details are given in Section II-C.) This representation allows the DAD model to be related directly to the general hybrid dynamical system model (1). The discrete states are 
$(q, z) \in Q$. The dynamical systems $\Sigma_{(q, z)}$ are defined by (7) and (8), with $f_{(q, z)}^{*}$ generating the continuous state dynamics. Each jump set $A_{(q, z)}$ is composed of conditions $y_{d, i}=0$ and $y_{e, j}=0$, where $y_{d}, y_{e}$ are given by $\varphi_{(q, z)}$. The general nature of $g_{q}$ and, hence, $\varphi_{(q, z)}$, allows arbitrarily complicated sets of event triggering conditions to be described for each $(q, z)$. The jump transition map $G_{(q, z)}$ is defined by the change in $q$ that corresponds to each $y_{d, i}=0$, along with the reset map (5) corresponding to each $y_{e, j}=0$.

Initial conditions for the model (2)-(6) are given by

$$
\begin{aligned}
& x\left(t_{0}\right)=x_{0} \\
& y\left(t_{0}\right)=y_{0} \\
& z\left(t_{0}\right)=z_{0}
\end{aligned}
$$

where $y_{0}$ is a solution of

$$
g\left(x_{0}, y_{0}, z_{0} ; \lambda\right)=0
$$

Note that in solving for $y_{0}$, the constraint switching described by (4) must be taken into account. Often $g=0$ will have multiple solutions. For a given $x_{0}, z_{0}$, and $\lambda$, there may be a number of possible $y_{0}$.

The following examples illustrate the DAD model structure (2)-(6). Even though they are quite simple, they exhibit many of the complexities associated with hybrid systems.

Example 1: This example is taken from [1]. The system is

$$
\dot{x}=A_{i} x
$$

where

$$
A_{1}=\left[\begin{array}{cc}
1 & -100 \\
10 & 1
\end{array}\right], \quad A_{2}=\left[\begin{array}{cc}
1 & 10 \\
-100 & 1
\end{array}\right] .
$$

The index $i$ changes from 1 to 2 when $x_{2}=2.75 x_{1}$ and from 2 to 1 when $x_{2}=0.36 x_{1}$. Initially $x_{0}=\left[\begin{array}{ll}0 & 1\end{array}\right]^{t}$ and $i=1$. The phase portrait and time response are shown in Figs. 1 and 2 , respectively.

This model can be rewritten in the DAD form as

$$
\begin{aligned}
& \dot{x}=\left[\begin{array}{cc}
1 & z_{1} \\
z_{2} & 1
\end{array}\right] x \\
& 0= \begin{cases}\lambda x_{1}-x_{2}-z_{3} y, & y<0 \\
x_{2}-0.36 x_{1}-z_{3} y, & y>0\end{cases} \\
& \left.\begin{array}{l}
z_{1}^{+}=z_{2}^{-} \\
z_{2}^{+}=z_{1}^{-} \\
z_{3}^{+}=-z_{3}^{-}
\end{array}\right\}, \quad \text { when } y=0
\end{aligned}
$$

where $x_{0}=\left[\begin{array}{ll}0 & 1\end{array}\right]^{t}, z_{0}=\left[\begin{array}{lll}-100 & 10 & 1\end{array}\right]^{t}, \lambda=2.75$, and hence, $y_{0}=-1$.

The change between $A_{1}$ and $A_{2}$ is achieved by resetting the matrix elements $z_{1}, z_{2}$ whenever a switching surface is encountered. The switching surfaces are given by the algebraic constraints. Alternating between active switching surfaces corresponds to flipping the sign of $z_{3}$.

The sensitivity of trajectories to variation of $\lambda$, i.e., the slope of the steeper switching surface, is presented in Section VI-A. $\square$

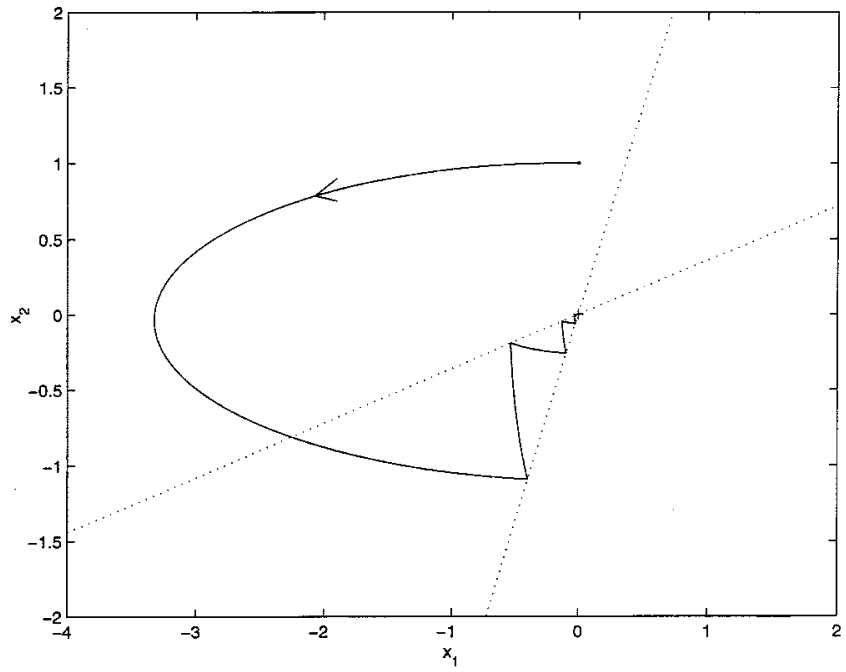

Fig. 1. Phase portrait for Example 1.

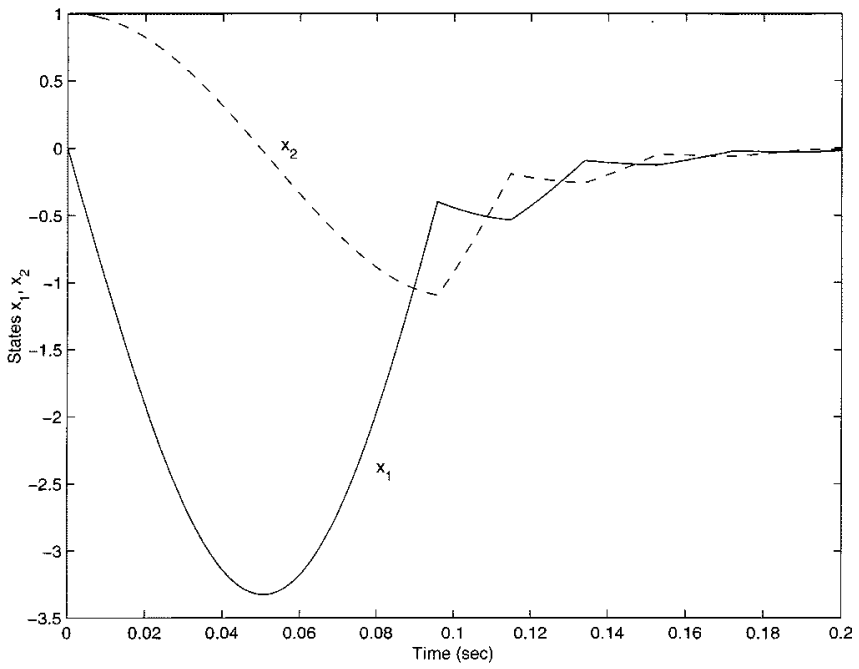

Fig. 2. Time-domain response for Example 1.

Example 2: This example is based on a case given in [21]. The system description is

$$
\begin{aligned}
& \dot{x}_{1}=x_{2} \\
& \dot{x}_{2}=-\operatorname{sgn}\left(x_{1}\right) \\
& x_{2}^{+}=0.8 x_{2}^{-}, \quad \text { when } x_{1}=0
\end{aligned}
$$

with $x_{0}=\left[\begin{array}{ll}0.25 & 0\end{array}\right]^{t}$. This is a crude model of the bounce of a ball when the coefficient of restitution is 0.8 . The phase portrait and time response of this system are shown in Figs. 3 and 4, respectively.

The model can be rewritten in the DAD form of (2)-(6) as

$$
\left.\begin{array}{rl}
\dot{x}_{1} & =y_{2} \\
\dot{x}_{2} & =z_{2} \\
0 & =x_{1}-y_{1} \\
0 & =x_{2}-z_{1}-y_{2} \\
z_{1}^{+} & =z_{1}^{-}+(1-\lambda) y_{2}^{-} \\
z_{2}^{+} & =-z_{2}^{-}
\end{array}\right\}, \quad \text { when } y_{1}=0
$$


where $x_{0}=\left[\begin{array}{ll}0.25 & 0\end{array}\right]^{t}, z_{0}=\left[\begin{array}{ll}0 & -1\end{array}\right]^{t}, \lambda=0.8$ and, hence, $y_{0}=\left[\begin{array}{ll}0.25 & 0\end{array}\right]^{t}$.

In establishing the DAD model structure (2)-(6), we made a deliberate decision not to allow resetting of the continuous states. Therefore, to implement the reset condition of this example, a discrete state $z_{1}$ and an algebraic state $y_{2}$ were introduced and $x_{2}$ was redefined to be continuous. The discrete state $z_{1}$ accumulates the step changes that occur at each reset event. The algebraic relationship between $z_{1}, y_{2}$, and the redefined $x_{2}$ ensures that $y_{2}$ replicates the behavior of the original (discontinuous) $x_{2}$.

The sensitivity of trajectories of this model to variation of $\lambda$, the coefficient of restitution, is explored in Section VI-B.

Trajectories of the DAD system (2)-(6) describe the behavior of the dynamic states $x$, the algebraic states $y$, and the discrete states $z$ over time. To formalize these concepts we define the flows of $x, y$, and $z$, respectively, as

$$
\begin{aligned}
& x(t)=\phi_{x}\left(x_{0}, z_{0}, t, \lambda\right) \\
& y(t)=\phi_{y}\left(x_{0}, z_{0}, t, \lambda\right) \\
& z(t)=\phi_{z}\left(x_{0}, z_{0}, t, \lambda\right)
\end{aligned}
$$

where

$$
\begin{gathered}
\frac{d}{d t}\left(\phi_{x}\left(x_{0}, z_{0}, t, \lambda\right)\right) \\
=f\left(\phi_{x}\left(x_{0}, z_{0}, t, \lambda\right), \phi_{y}\left(x_{0}, z_{0}, t, \lambda\right),\right. \\
\left.\quad \phi_{z}\left(x_{0}, z_{0}, t, \lambda\right) ; \lambda\right) \\
0=g\left(\phi_{x}\left(x_{0}, z_{0}, t, \lambda\right), \phi_{y}\left(x_{0}, z_{0}, t, \lambda\right),\right. \\
\left.\phi_{z}\left(x_{0}, z_{0}, t, \lambda\right) ; \lambda\right)
\end{gathered}
$$

and $\phi_{z}\left(x_{0}, z_{0}, t, \lambda\right)$ is piecewise constant with step transitions between the constant sections described by the reset equations (5).

From the definitions of the flows, it is clear that

$$
\begin{aligned}
& \phi_{x}\left(x_{0}, z_{0}, t_{0}, \lambda\right)=x_{0} \\
& \phi_{y}\left(x_{0}, z_{0}, t_{0}, \lambda\right)=y_{0} \\
& \phi_{z}\left(x_{0}, z_{0}, t_{0}, \lambda\right)=z_{0} .
\end{aligned}
$$

Notice that $\phi_{y}$ has been defined in terms of $x_{0}$ and $z_{0}$ rather than $y_{0}$. This reflects the dependence of $y_{0}$ on $x_{0}, z_{0}$, and $\lambda$, as described by (12). Therefore the definitions of $\phi_{x}, \phi_{y}$, and $\phi_{z}$ establish the dependence of the flows on $x_{0}, z_{0}$, and $\lambda$.

It is clear that the notation can quickly become unwieldy. Therefore in the sequel we will generally write the model more compactly as

$$
\begin{aligned}
& \underline{\dot{x}}=\underline{f}(\underline{x}, y) \\
& 0=g^{(0)}(\underline{x}, y) \\
& 0=\left\{\begin{array}{ll}
g^{(i-)}(\underline{x}, y) & y_{d, i}<0, \\
g^{(i+)}(\underline{x}, y) & y_{d, i}>0,
\end{array} \quad i=1, \cdots, d\right. \\
& \underline{x}^{+}=\underline{h}_{j}\left(\underline{x}^{-}, y^{-}\right) \quad y_{e, j}=0, \quad j \in\{1, \cdots, e\}(16)
\end{aligned}
$$

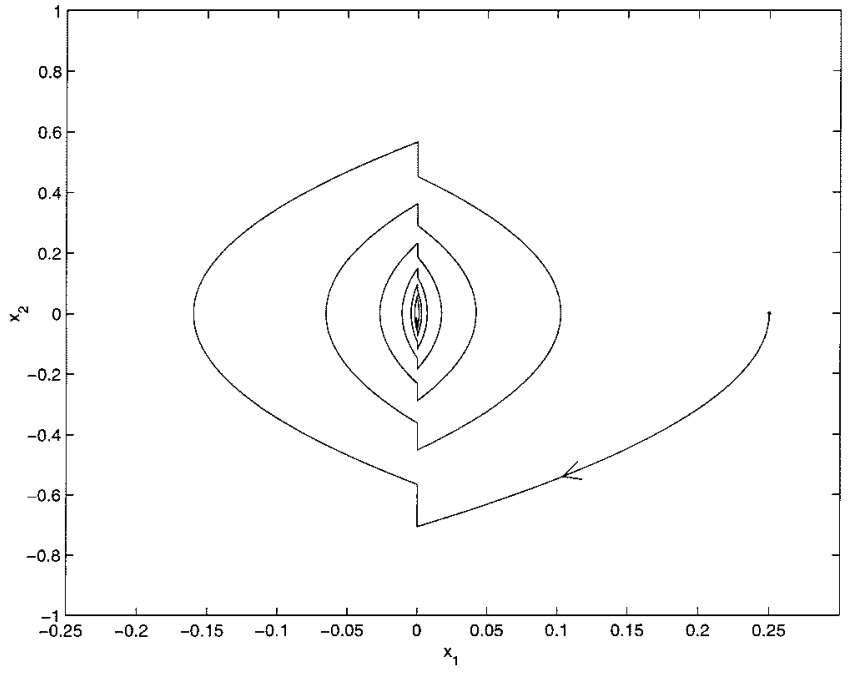

Fig. 3. Phase portrait for Example 2.

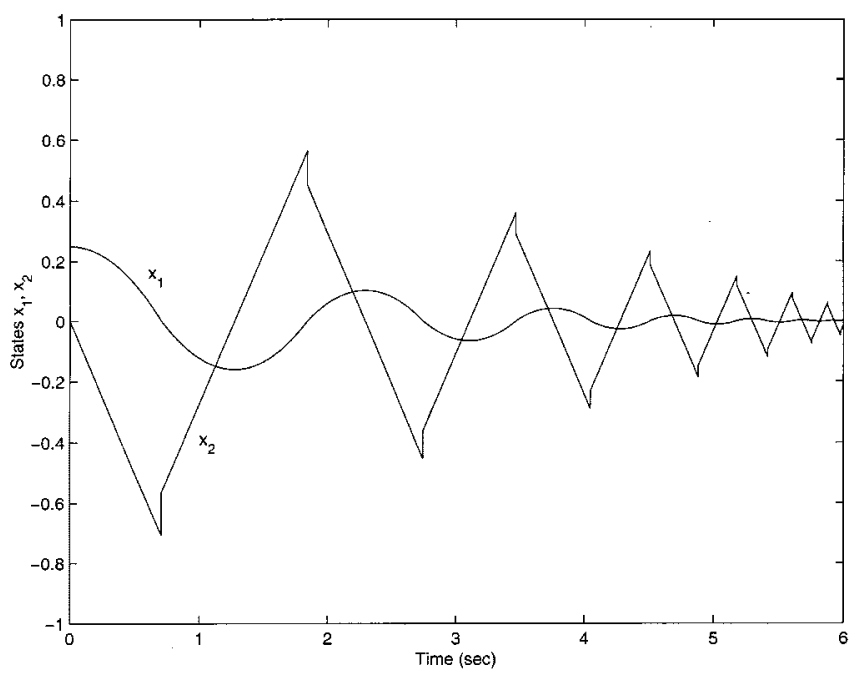

Fig. 4. Time-domain response for Example 2.

where

$$
\begin{aligned}
& \underline{x}=\left[\begin{array}{l}
x \\
z \\
\lambda
\end{array}\right] \in \underline{X}=X \times Z \times L \subseteq \mathbb{R}^{\underline{n}=n+l+p} \\
& \underline{f}=\left[\begin{array}{c}
f \\
0 \\
0
\end{array}\right] \quad \underline{h}_{j}=\left[\begin{array}{c}
x \\
h_{j} \\
\lambda
\end{array}\right] .
\end{aligned}
$$

The system flow is defined accordingly as

$$
\phi\left(\underline{x}_{0}, t\right)=\left[\begin{array}{l}
\phi_{\underline{x}}\left(\underline{x}_{0}, t\right) \\
\phi_{y}\left(\underline{x}_{0}, t\right)
\end{array}\right]=\left[\begin{array}{l}
\underline{x}(t) \\
y(t)
\end{array}\right] .
$$

Let the times at which events occur be given by $\left\{\tau_{k}: t_{0}<\tau_{1}<\right.$ $\left.\tau_{2}<\cdots\right\}$.

Notice that the definition of $f$ ensures that $z$ and $\lambda$ remain constant away from reset events (16). Further, $\underline{h}_{j}$ ensures that $x$ and $\lambda$ remain unchanged at a reset event. As with (7) and (8), over each of the open time intervals $\left(\tau_{k}, \tau_{k+1}\right)$ the system is described by a smooth DA model

$$
\underline{\dot{x}}=\underline{f}(\underline{x}, y)
$$




$$
0=g(\underline{x}, y)
$$

where $g$ is composed of (14) together with functions from (15) chosen depending on the signs of the elements of $y_{d}$. [Recall that the definition of the $\tau_{k}$ ensures that no elements of $y_{d}$ can change sign during the period $\left(\tau_{k}, \tau_{k+1}\right)$.]

Trajectory sensitivity analysis considers the influence of (small) variations in the initial conditions $x_{0}, z_{0}$ and parameters $\lambda$, (or equivalently $\underline{x}_{0}$ ) on the flow $\phi$. Sensitivity relationships are developed in Sections III-V.

\section{Properties of DA Systems}

Before focusing on sensitivities, it is important to establish some basic properties of DA systems. This section provides a brief summary. A much more extensive investigation is presented in [22].

Consider the DA model (18) and (19) which describes behavior between events, say during the open interval $\left(\tau_{k}, \tau_{k+1}\right)$. The algebraic equations define the constraint surface or constraint set

$$
\mathcal{C}=\{(\underline{x}, y) \in \underline{X} \times Y: g(\underline{x}, y)=0\} .
$$

The system is constrained to lie on this surface (in the set $\mathcal{C}$ ) over the period $\left(\tau_{k}, \tau_{k+1}\right)$. Equation (19) ensures that flows satisfy the constraints. The differential equations (DE's) drive the flows over the constraint surface.

As indicated earlier, the implicit function theorem can be used to establish a connection between the DA model and a locally equivalent DE model. If $g_{y} \equiv(\partial g / \partial y)$ is nonsingular at a point $\left(\underline{x}^{*}, y^{*}\right) \in \mathcal{C}$, then it follows from the implicit function theorem that there exist neighborhoods $U_{\underline{x}} \subset \mathbb{R} \underline{\underline{n}}$ of $\underline{x}^{*}$ and $U_{y} \subset \mathbb{R}^{m}$ of $y^{*}$ and a vector function $\varphi: U_{\underline{x}} \longrightarrow U_{y}$ such that

$$
y^{*}=\varphi\left(\underline{x}^{*}\right)
$$

and

$$
0=g(\underline{x}, \varphi(\underline{x})), \quad \forall \underline{x} \in U_{\underline{x}} .
$$

The function $\varphi$ is unique in the sense that if $\underline{x} \in U_{\underline{x}}, y \in U_{y}$, and $g(\underline{x}, y)=0$ then

$$
y=\varphi(\underline{x}) .
$$

Upon substitution of (21) into (18), the DA system reduces to

$$
\begin{aligned}
\underline{\dot{x}} & =\underline{f}(\underline{x}, \varphi(\underline{x})) \\
& =\underline{f}^{*}(\underline{x})
\end{aligned}
$$

i.e., a local DE description of the system. This local result is valid wherever $g_{y}$ is nonsingular.

The algebraic equations $g=0$ may have more than one solution. For a given $\underline{x}^{*}$, there may be a number of $y_{i}^{*}$ such that $\left(\underline{x}^{*}, y_{i}^{*}\right) \in \mathcal{C}$. At each point $\left(\underline{x}^{*}, y_{i}^{*}\right)$ where $g_{y}$ is nonsingular, the implicit function theorem guarantees the existence of a unique (in the sense defined above) vector function $\varphi_{i}(\underline{x})$. A local DE description

$$
\underline{\dot{x}}=\underline{f}_{i}^{*}(\underline{x})
$$

corresponds to each $\varphi_{i}$.
At points where $g_{y}$ is singular, the model may break down. The conditions of the implicit function theorem are no longer satisfied, so there is no guarantee of the existence of a welldefined relationship (21) between $\underline{x}$ and $y$. In general, a system trajectory cannot continue through a point where $g_{y}$ is singular. Such singular points are therefore known as impasse points and the set of points

$$
\mathcal{I}=\left\{(\underline{x}, y) \in \mathcal{C}: \operatorname{det} g_{y}(\underline{x}, y)=0\right\}
$$

is called the impasse surface [22], [23].

The set $\mathcal{C} \backslash \mathcal{I}$ contains all points on the constraint surface which are not impasse points. Therefore, the DA model is well defined in $\mathcal{C} \backslash \mathcal{I}$. This set defines an $(n+l+p)$-dimensional manifold [22], and has been called the causal region. It is generally not a connected set though, with different components of the set corresponding to different $\varphi_{i}$.

In the development and use of trajectory sensitivities, we will be focusing upon cases where model breakdown is not a concern. We therefore make the following assumption.

Assumption 1: All trajectories remain within the causal region $\mathcal{C} \backslash \mathcal{I}$, i.e., $g_{y}$ is nonsingular along trajectories.

Because Assumption 1 ensures that algebraic singularity is avoided, the type of jump behavior discussed in [24] cannot occur. The jumps which are a feature of the DAD model occur only in response to discrete events.

\section{TRAJECTORY SENSITIVITy ANALYSIS}

The flow $\phi$ of a system will generally vary with changes in parameters and/or initial conditions. Trajectory sensitivity analysis provides a way of quantifying the changes in the flow that result from (small) changes in parameters and initial conditions. The development of these sensitivity concepts will be based upon the compact form of the DAD model (13)-(16). Recall that in this model, $\underline{x}_{0}$ incorporates the initial conditions $x_{0}$ and $z_{0}$, as well as the parameters $\lambda$. Therefore, the sensitivity of the flow to $\underline{x}_{0}$ fully describes its sensitivity to $x_{0}, z_{0}$, and $\lambda$.

In Section II-B, we defined the system flow $\phi$ in terms of $\underline{x}_{0}$. The dependence of $\phi$ on $y_{0}$ is not explicit, but follows from (12). Therefore, in determining trajectory sensitivities, we will not directly establish the sensitivity of flows to changes in $y_{0}$. Rather, such sensitivity is given implicitly by sensitivity to $\underline{x}_{0}$.

Trajectory sensitivities follow from a Taylor series expansion of the flows $\phi_{\underline{x}}$ and $\phi_{y}$. Referring to (17), the expansion for $\phi_{\underline{x}}$ can be expressed as

$$
\begin{aligned}
\Delta \underline{x}(t) & =\Delta \phi_{\underline{x}}\left(\underline{x}_{0}, t\right) \\
& =\frac{\partial \phi_{\underline{x}}\left(\underline{x}_{0}, t\right)}{\partial \underline{x}_{0}} \Delta \underline{x}_{0}+\text { higher order terms. }
\end{aligned}
$$

Neglecting the higher order terms and using (17), we obtain

$$
\begin{aligned}
\Delta \underline{x}(t) & \approx \frac{\partial \underline{x}(t)}{\partial \underline{x}_{0}} \Delta \underline{x}_{0} \\
& \equiv \underline{x}_{\underline{x}_{0}}(t) \Delta \underline{x}_{0}
\end{aligned}
$$

where $\underline{x}_{\underline{x}_{0}} \in \mathbb{R} \underline{n} \times \underline{n}$. From (24), the sensitivity of the flow $\phi_{\underline{x}}$ to (small) changes $\Delta \underline{x}_{0}$ is given by the trajectory sensitivities $\underline{x_{x_{0}}}(t)$. 
A similar Taylor series expansion of $\phi_{y}$ yields

$$
\begin{aligned}
\Delta y(t) & =\Delta \phi_{y}\left(\underline{x}_{0}, t\right) \\
& =\frac{\partial \phi_{y}\left(\underline{x}_{0}, t\right)}{\partial \underline{x}_{0}} \Delta \underline{x}_{0}+\text { higher order terms } .
\end{aligned}
$$

Again neglecting the higher order terms and using (17) results in

$$
\begin{aligned}
\Delta y(t) & \approx \frac{\partial y(t)}{\partial \underline{x}_{0}} \Delta \underline{x}_{0} \\
& \equiv \underline{y}_{x_{0}}(t) \Delta \underline{x}_{0}
\end{aligned}
$$

where $y_{\underline{x}_{0}} \in \mathbb{R}^{m \times \underline{n}}$. In this case, the sensitivity of the flow $\phi_{y}$ to (small) changes $\Delta \underline{x}_{0}$ is given by the trajectory sensitivities $y_{\underline{x}_{0}}(t)$.

Once the trajectory sensitivities $\underline{x}_{\underline{x}_{0}}(t)$ and $y_{\underline{x}_{0}}(t)$ are known, the sensitivity of the system flow $\bar{\phi}$ to small changes in initial conditions and parameters, which are described by $\Delta \underline{x}_{0}$, can be determined from

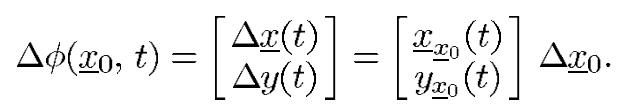

We have yet to consider the calculation of the trajectory sensitivities. Details are provided in the following sections.

\section{SENSITIVITy EVOLUTION AWAY FROM EVENTS}

In this section we discuss the calculation of the trajectory sensitivities $\underline{x}_{\underline{x}_{0}}(t)$ and $y_{\underline{x}_{0}}(t)$ over the open time intervals $\left(\tau_{k}, \tau_{k+1}\right)$, i.e., away from events. The behavior of sensitivities at switching and reset events is presented in Section V.

Away from events, the system model is given by (18) and (19). Differentiating this DA system with respect to the initial conditions $\underline{x}_{0}$ results in

$$
\begin{aligned}
\underline{\dot{x}}_{\underline{x}_{0}} & =\underline{f}_{\underline{x}}(t) \underline{x}_{\underline{x}_{0}}+\underline{f}_{y}(t) y_{\underline{x}_{0}} \\
0 & =g_{\underline{x}}(t) \underline{x}_{\underline{x}_{0}}+g_{y}(t) \underline{y}_{\underline{x}_{0}}
\end{aligned} .
$$

Note that $\underline{f_{x}}, \underline{f_{y}}, g_{\underline{x}}, g_{y}$ are evaluated along the flow $\phi\left(\underline{x}_{0}, t\right)$ and, hence, are time-varying matrices.

Initial conditions for $\underline{x}_{\underline{x}_{0}}$ on the first time interval $\left[t_{0}, \tau_{1}\right)$ are obtained by differentiating (9) and (11) with respect to $\underline{x}_{0}$

$$
\underline{x}_{\underline{x}_{0}}\left(t_{0}\right)=I
$$

where $I$ is the identity matrix. Initial conditions for $y_{\underline{x}_{0}}$ follow from (28)

$$
0=g_{\underline{x}}\left(t_{0}\right) \underline{x}_{\underline{x}_{0}}\left(t_{0}\right)+g_{y}\left(t_{0}\right) y_{\underline{x}_{0}}\left(t_{0}\right) .
$$

In accordance with Assumption 1, $g_{y}\left(t_{0}\right)$ is nonsingular so (29) can be used to obtain

$$
y_{\underline{x}_{0}}\left(t_{0}\right)=-\left(g_{y}\left(t_{0}\right)\right)^{-1} g_{\underline{x}}\left(t_{0}\right) .
$$

On other time intervals, say $\left(\tau_{k}, \tau_{k+1}\right)$, the initial sensitivities $\underline{x}_{\underline{x}_{0}}\left(\tau_{k}^{+}\right), y_{\underline{x}_{0}}\left(\tau_{k}^{+}\right)$are given by the jump conditions described in Section $\overline{\mathrm{V}}$.
An equivalent DE model for calculating $\underline{x}_{x_{0}}(t)$ can be obtained by rearranging (28), taking account of Assumption 1, to give

$$
y_{\underline{x}_{0}}=-g_{y}^{-1} g_{\underline{x}} \underline{x}_{\underline{x}_{0}} .
$$

Substituting into (27) results in

$$
\underline{\dot{x}}_{\underline{x}_{0}}=\left(\underline{f_{x}}-\underline{f}_{y} g_{y}^{-1} g_{\underline{x}}\right) \underline{x}_{\underline{x}_{0}} .
$$

This equation describes the sensitivity of trajectories of the reduced DE model given by (22). Given $\underline{x}_{\underline{x}_{0}}$, we can obtain $y_{\underline{x}_{0}}$ from (31), keeping in mind that $g_{\underline{x}}$ and $g_{y}$ are time-varying matrices. [Note though that (31) and (32) are not normally used in the computation of sensitivities. It is more efficient to solve (27) and (28) simultaneously. Details are given in Section VII.]

Equation (31) highlights the importance of Assumption 1. If $g_{y}$ is nearly singular, corresponding to the trajectory being close to the impasse surface, $y_{\underline{x}_{0}}$ can become large, even for small $\underline{x}_{x_{0}}$. Under such conditions, a variation in initial conditions or parameters which causes only a small change in the dynamic states $x$ and $z$ may induce a large change in the algebraic states $y$.

The compact form of the model (13)-(16) is convenient for developing the trajectory sensitivity equations. However it can be helpful to revert to the full model description to examine the sensitivity of the states $x$ and $z$ to their initial conditions and to the parameters $\lambda$. Expanding (27) results in

$$
\begin{aligned}
\frac{d}{d t}\left[\begin{array}{l}
x_{\underline{x}_{0}} \\
z_{\underline{x}_{0}} \\
\lambda_{\underline{x}_{0}}
\end{array}\right]= & \frac{d}{d t}\left[\begin{array}{ccc}
x_{x_{0}} & x_{z_{0}} & x_{\lambda} \\
z_{x_{0}} & z_{z_{0}} & z_{\lambda} \\
\lambda_{x_{0}} & \lambda_{z_{0}} & \lambda_{\lambda}
\end{array}\right] \\
= & {\left[\begin{array}{cccc}
f_{x} & f_{z} & f_{\lambda} \\
0 & 0 & 0 \\
0 & 0 & 0
\end{array}\right]\left[\begin{array}{ccc}
x_{x_{0}} & x_{z_{0}} & x_{\lambda} \\
z_{x_{0}} & z_{z_{0}} & z_{\lambda} \\
\lambda_{x_{0}} & \lambda_{z_{0}} & \lambda_{\lambda}
\end{array}\right] } \\
& +\left[\begin{array}{c}
f_{y} \\
0 \\
0
\end{array}\right]\left[\begin{array}{lll}
y_{x_{0}} & y_{z_{0}} & y_{\lambda}
\end{array}\right] .
\end{aligned}
$$

Initial conditions follow from (29)

$$
\begin{array}{lll}
x_{x_{0}}\left(t_{0}\right)=I & x_{z_{0}}\left(t_{0}\right)=0 & x_{\lambda}\left(t_{0}\right)=0 \\
z_{x_{0}}\left(t_{0}\right)=0 & z_{z_{0}}\left(t_{0}\right)=I & z_{\lambda}\left(t_{0}\right)=0 \\
\lambda_{x_{0}}\left(t_{0}\right)=0 & \lambda_{z_{0}}\left(t_{0}\right)=0 & \lambda_{\lambda}\left(t_{0}\right)=I .
\end{array}
$$

It can be seen from (33) that the sensitivities $z_{\underline{x}_{0}}$ and $\lambda_{\underline{x}_{0}}$ remain constant over the intervals between events. However the evolution of $x_{\underline{x}_{0}}$ is influenced by all the $\underline{x}_{\underline{x}_{0}}$ and $y_{\underline{x}_{0}}$ sensitivities.

\section{SENSitivity Behavior at Events}

\section{A. Preliminary Concepts}

In Section IV, we established (27) and (28) describing the evolution of the sensitivities $\underline{x}_{\underline{x}_{0}}$ and $y_{\underline{x}_{0}}$ over the intervals between switching and reset events. To fully describe the sensitivities though, we must quantify their behavior at these discrete events that are characteristic of hybrid systems. To determine 
this behavior, we will consider the system at a single event. Accordingly, attention is focused on the model

$$
\begin{array}{rlrl}
\underline{\dot{x}} & =\underline{f}(\underline{x}, y) \\
0 & = \begin{cases}g^{-}(\underline{x}, y), & s(\underline{x}, y)<0 \\
g^{+}(\underline{x}, y), & s(\underline{x}, y)>0\end{cases} \\
\underline{x}^{+} & =\underline{h}\left(\underline{x}^{-}, y^{-}\right), & s(\underline{x}, y)=0
\end{array}
$$

which is directly related to the compact DAD model (13)-(16). A number of comments should be made about this model.

- In this model, the switching and reset events are triggered by the condition $s(\underline{x}, y)=0$ rather than by an element of $y$ passing through zero. This modification helps to identify the role of the triggering condition. We will later revert to the situation where the event is described by a condition $y_{k}=0$.

- Notice that both switching and state resetting occur when $s(\underline{x}, y)=0$. This is the most general case. Sensitivity behavior at independent switching and reset events follows from this general case and will be discussed later.

- We are investigating a single event. However the extension to the usual case where there are multiple events, each separated by a finite time interval, is straightforward.

- Coincident events, where two triggering conditions $s_{1}=0$ and $s_{2}=0$ are simultaneously satisfied, are nongeneric. However the analysis can be extended to such a case. This is considered at the end of Section V-B.

Define the triggering hypersurface as

$$
\mathcal{S}=\{(\underline{x}, y) \in \mathcal{C} \backslash \mathcal{I}: s(\underline{x}, y)=0\} .
$$

We are interested in the sensitivity of trajectories which pass through $\mathcal{S}$. It is convenient to assume that the trajectory starts from a point where $s(\underline{x}, y)<0$ passes through $\mathcal{S}$, and proceeds to a point where $s(\underline{x}, y)>0$. There is no loss of generality in this assumption. Let $\phi\left(\underline{x}_{0}, t\right)=\left[\underline{x}(t)^{t} y(t)^{t}\right]^{t}$ be such a trajectory, which starts from $\phi\left(\underline{x}_{0}, t_{0}\right)=\left[\begin{array}{ll}\underline{x}_{0}{ }^{t} & y_{0}^{t}\end{array}\right]^{t}$, intersects $\mathcal{S}$ at the point $\phi\left(\underline{x}_{0}, \tau\right)=\left[\underline{x}(\tau)^{t} y(\tau)^{t}\right]^{t}$, and proceeds to the point $\phi\left(\underline{x}_{0}, t_{1}\right)=\left[\begin{array}{ll}x_{1}^{t} & y_{1}^{t}\end{array}\right]^{t}$. The intersection point $(\underline{x}(\tau), y(\tau))$ is called the junction point and $\tau$ is called the junction time. We define time $\tau^{-}<\tau$ to be the limit as $t$ approaches $\tau$ from below and $\tau^{+}>\tau$ as the limit as $\tau$ is approached from above.

The concept of trajectories passing through $\mathcal{S}$ is important. Sensitivities cannot be defined for trajectories which are tangential to $\mathcal{S}$. Consider such a trajectory. Then there exists an incremental change in the initial conditions $\underline{x}_{0}$ such that the intersection point disappears. But for a different small change in $\underline{x}_{0}$ the intersection point persists. Therefore, at the tangent point the trajectory is infinitely sensitive to initial conditions. To overcome this difficulty we make the following assumption.

Assumption 2: Trajectories are transversal to the triggering hypersurface $\mathcal{S}$.

It is also necessary to make the next assumption.

Assumption 3: The triggering function $s(\underline{x}, y)$ has a unique normal $\nabla s(\underline{x}, y)$ at points in $\mathcal{S}$.

These assumptions are commonly made in the analysis of hybrid systems (for example, see [17, Assumption 5.3]). They are also closely related to the DEDS concept of deterministic similarity which underlies infinitesimal perturbation analysis [11].

The transversality condition ensures that trajectories must cross $\mathcal{S}$, i.e., they cannot be tangential to $\mathcal{S}$. The condition is satisfied at a junction point $(\underline{x}(\tau), y(\tau))$ if

$$
\nabla s(\underline{x}(\tau), y(\tau))^{t}[\underline{\dot{x}}(\tau)] \neq 0
$$

For the trajectory approaching $\mathcal{S}$, we have that at time $\tau^{-}<\tau$

$$
\underline{\dot{x}}\left(\tau^{-}\right)=\underline{f}\left(\underline{x}\left(\tau^{-}\right), y^{-}\left(\tau^{-}\right)\right)
$$

where $y^{-}\left(\tau^{-}\right)$is given by $g^{-}\left(\underline{x}\left(\tau^{-}\right), y^{-}\left(\tau^{-}\right)\right)=0$. Also, by differentiating $g^{-}$we obtain

$$
\begin{aligned}
\dot{y}\left(\tau^{-}\right) & =-\left.\left(\left(g_{y}^{-}\right)^{-1} g_{\underline{x}}^{-}\right)\right|_{\tau^{-}} \underline{\dot{x}}\left(\tau^{-}\right) \\
& =-\left.\left(\left(g_{y}^{-}\right)^{-1} g_{\underline{x}}^{-}\right)\right|_{\tau^{-}} \underline{f}\left(\underline{x}\left(\tau^{-}\right), y^{-}\left(\tau^{-}\right)\right) .
\end{aligned}
$$

At the limit $\tau^{-}$, the transversality condition (37) becomes

$$
\begin{aligned}
& \frac{\partial s}{\partial \underline{x}} \underline{\dot{x}}(\tau)+\frac{\partial s}{\partial y} \dot{y}(\tau) \\
& \quad=\left.\left(s_{\underline{x}}-s_{y}\left(g_{y}^{-}\right)^{-1} g_{\underline{x}}^{-}\right)\right|_{\tau^{-}} \underline{f}\left(\underline{x}\left(\tau^{-}\right), y^{-}\left(\tau^{-}\right)\right) \\
& \quad \neq 0 .
\end{aligned}
$$

The transversality condition of Assumption 2 ensures that the junction point depends continuously on initial conditions $\underline{x}_{0}$ [8].

We also need to ensure that the switching and reset events are consistent with $s(\underline{x}, y)$ changing sign as $\mathcal{S}$ is crossed. The following assumption is therefore made.

Assumption 4: At a junction point $(\underline{x}(\tau), y(\tau)) \in \mathcal{S}$, $s\left(\underline{x}\left(\tau^{-}\right), y\left(\tau^{-}\right)\right) \times s\left(\underline{x}\left(\tau^{+}\right), y\left(\tau^{+}\right)\right)<0$.

This assumption is commonly made to ensure triggering events do not accumulate [17]. It is generically satisfied for realistic systems. If it was not satisfied, then the trajectory could reach an impasse at the triggering hypersurface. Upon encountering $\mathcal{S}$, the algebraic equations would switch from $g^{-}$ to $g^{+}$. However if $s\left(\underline{x}\left(\tau^{+}\right), y\left(\tau^{+}\right)\right) \ngtr 0$, the model would be forced to switch back to $g^{-}$, which may result in switching to $g^{+}$again, and so on.

\section{B. Jump Conditions}

We now proceed to develop the conditions which govern the behavior of trajectory sensitivities at discrete events. First, the dependence of the junction time $\tau$ on $\underline{x}_{0}$ will be established. That is then used to obtain the desired sensitivity jump conditions.

Based on the model presented in Section $\mathrm{V}-\mathrm{A}$, at time $\tau^{-}<\tau$

$$
\begin{aligned}
& \underline{x}^{-}=\underline{x}\left(\tau^{-}\right)=\phi_{\underline{x}}\left(\underline{x}_{0}, \tau^{-}\right) \\
& y^{-}=y^{-}\left(\tau^{-}\right)=\phi_{y}\left(\underline{x}_{0}, \tau^{-}\right)
\end{aligned}
$$

where

$$
0=g^{-}\left(\underline{x}^{-}, y^{-}\right)
$$

Also, in the limit

$$
s\left(\underline{x}^{-}, y^{-}\right)=0 .
$$


From (42) it follows that

$$
s_{\underline{x}} d \underline{x}^{-}+s_{y} d y^{-}=0
$$

and from (41)

$$
g_{\underline{x}}^{-} d \underline{x}^{-}+g_{y}^{-} d y^{-}=0
$$

where all partial derivatives are evaluated at $\tau^{-}$. Rearranging (44), taking account of Assumption 1, and substituting into (43) results in

$$
\left.\left(s_{\underline{x}}-s_{y}\left(g_{y}^{-}\right)^{-1} g_{\underline{x}}^{-}\right)\right|_{\tau^{-}} d \underline{x}^{-}=0 .
$$

Now from (39)

$$
\frac{d \underline{x}^{-}}{d \underline{x}_{0}}=\left.\left(\frac{\partial \underline{x}}{\partial \underline{x}_{0}}+\frac{\partial \underline{x}}{\partial \tau} \frac{d \tau}{d \underline{x}_{0}}\right)\right|_{\tau^{-}} .
$$

Also, (34) gives

$$
\left.\frac{\partial \underline{x}}{\partial \tau}\right|_{\tau^{-}}=\underline{\dot{x}}\left(\tau^{-}\right)=\underline{f}\left(\underline{x}\left(\tau^{-}\right), y^{-}\left(\tau^{-}\right)\right) .
$$

Therefore, substituting (46) and (47) into (45) yields

$$
\begin{aligned}
& \left.\left(s_{\underline{x}}-s_{y}\left(g_{y}^{-}\right)^{-1} g_{\underline{x}}^{-}\right)\right|_{\tau^{-}} \\
& \cdot\left(\underline{x}_{x_{0}}\left(\tau^{-}\right)+\underline{f}\left(\underline{x}\left(\tau^{-}\right), y^{-}\left(\tau^{-}\right)\right) \frac{d \tau}{d \underline{x}_{0}}\left(\tau^{-}\right)\right)=0 .
\end{aligned}
$$

Rearranging gives

$$
\begin{aligned}
\frac{d \tau}{d \underline{x}_{0}}\left(\tau^{-}\right) & =\tau_{\underline{x}_{0}} \\
& =-\frac{\left.\left(s_{\underline{x}}-s_{y}\left(g_{\bar{y}}^{-}\right)^{-1} g_{\underline{x}}^{-}\right)\right|_{\tau^{-}} \underline{x}_{\underline{x}_{0}}\left(\tau^{-}\right)}{\left.\left(s_{\underline{x}}-s_{y}\left(g_{\bar{y}}^{-}\right)^{-1} g_{\underline{x}}^{-}\right)\right|_{\tau^{-}} \underline{f}\left(\underline{x}\left(\tau^{-}\right), y^{-}\left(\tau^{-}\right)\right)} .
\end{aligned}
$$

Recall that $\tau$ is the time at which the event is triggered and so is not influenced by the actual event that is subsequently triggered. Therefore $\tau_{\underline{x}_{0}}$ is also independent of the triggered event.

Notice that if the transversality condition (38) is satisfied, then the denominator in (48) will be nonzero. Conversely, if the transversality condition is not satisfied, then (48) breaks down and the junction time $\tau$ becomes infinitely sensitive to initial conditions $\underline{x}_{0}$. This behavior reflects the discussion which preceded Assumption 2.

We now proceed to determine the jump conditions describing the behavior of the trajectory sensitivities at the discrete event. Referring to (46), we know that

$$
\frac{d \underline{x}}{d \underline{x}_{0}}\left(\tau^{-}\right)=\underline{x}_{\underline{x}_{0}}\left(\tau^{-}\right)+\left.\frac{\partial \underline{x}}{\partial \tau}\right|_{\tau^{-}} \tau_{\underline{x}_{0}} .
$$

Substituting in (47) and rearranging gives

$$
\underline{x}_{\underline{x}_{0}}\left(\tau^{-}\right)=\frac{d \underline{x}}{d \underline{x}_{0}}\left(\tau^{-}\right)-\underline{f}\left(\underline{x}\left(\tau^{-}\right), y^{-}\left(\tau^{-}\right)\right) \tau_{\underline{x}_{0}} .
$$

Similarly, we obtain at $\tau^{+}$

$$
\frac{d \underline{x}}{d \underline{x}_{0}}\left(\tau^{+}\right)=\underline{x}_{\underline{x}_{0}}\left(\tau^{+}\right)+\left.\frac{\partial \underline{x}}{\partial \tau}\right|_{\tau^{+}} \tau_{\underline{x}_{0}} .
$$

In this case

$$
\left.\frac{\partial \underline{x}}{\partial \tau}\right|_{\tau^{+}}=\underline{\dot{x}}\left(\tau^{+}\right)=\underline{f}\left(\underline{x}\left(\tau^{+}\right), y^{+}\left(\tau^{+}\right)\right)
$$

where now $\underline{x}\left(\tau^{+}\right)$and $y^{+}\left(\tau^{+}\right)$satisfy

$$
g^{+}\left(\underline{x}\left(\tau^{+}\right), y^{+}\left(\tau^{+}\right)\right)=0 .
$$

Hence

$$
\underline{x}_{\underline{x}_{0}}\left(\tau^{+}\right)=\frac{d \underline{x}}{d \underline{x}_{0}}\left(\tau^{+}\right)-\underline{f}\left(\underline{x}\left(\tau^{+}\right), y^{+}\left(\tau^{+}\right)\right) \tau_{\underline{x}_{0}} .
$$

The jump condition in $\underline{x}_{x_{0}}$ is given by (50) and (53). But first we must use (36), which gives

$$
\frac{d \underline{x}^{+}}{d \underline{x}_{0}}=\frac{\partial \underline{h}}{\partial \underline{x}_{x}} \frac{d \underline{x}^{-}}{d \underline{x}_{0}}+\frac{\partial \underline{h}}{\partial y} \frac{d y^{-}}{d \underline{x}_{0}}
$$

i.e.,

$$
\frac{d \underline{x}}{d \underline{x}_{0}}\left(\tau^{+}\right)=\left.\left(\underline{h}_{\underline{x}} \frac{d \underline{x}}{d \underline{x}_{0}}+\underline{h}_{y} \frac{d y}{d \underline{x}_{0}}\right)\right|_{\tau^{-}} .
$$

Using (44) yields

$$
\begin{aligned}
\frac{d \underline{x}}{d \underline{x}_{0}}\left(\tau^{+}\right) & =\left.\left(\underline{h}_{\underline{x}}-\underline{h}_{y}\left(g_{y}^{-}\right)^{-1} g_{\underline{x}^{-}}\right)\right|_{\tau^{-}} \frac{d \underline{x}}{d \underline{x}_{0}}\left(\tau^{-}\right) \\
& =\underline{h}_{\underline{x}}^{*} \frac{d \underline{x}}{d \underline{x}_{0}}\left(\tau^{-}\right) .
\end{aligned}
$$

Subtracting (50) from (53) and using (55) results in the jump condition

$$
\begin{aligned}
& \underline{x}_{x_{0}}\left(\tau^{+}\right)-\underline{x}_{x_{0}}\left(\tau^{-}\right) \\
& =\underline{h}_{x}^{*} \frac{d \underline{x}}{d \underline{x}_{0}}\left(\tau^{-}\right)-\frac{d \underline{x}}{d \underline{x}_{0}}\left(\tau^{-}\right)-\left(\underline{f}^{+}-\underline{f}^{-}\right) \tau_{\underline{x}_{0}} \\
& =\left(\underline{h}_{\underline{x}}^{*}-I\right) \frac{d \underline{x}}{d \underline{x}_{0}}\left(\tau^{-}\right)-\left(\underline{f}^{+}-\underline{f}^{-}\right) \tau_{\underline{x}_{0}}
\end{aligned}
$$

where for clarity we have adopted the notation

$$
\begin{aligned}
& \underline{f}^{-} \equiv \underline{f}\left(\underline{x}\left(\tau^{-}\right), y^{-}\left(\tau^{-}\right)\right) \\
& \underline{f}^{+} \equiv \underline{f}\left(\underline{x}\left(\tau^{+}\right), y^{+}\left(\tau^{+}\right)\right) .
\end{aligned}
$$

Reusing (50) to eliminate $\left(d \underline{x} / d \underline{x}_{0}\right)\left(\tau^{-}\right)$gives

$$
\begin{aligned}
& \underline{x}_{\underline{x}_{0}}\left(\tau^{+}\right)-\underline{x}_{\underline{x}_{0}}\left(\tau^{-}\right) \\
& \quad=\left(\underline{h}_{\underline{x}}^{*}-I\right)\left(\underline{x}_{\underline{x}_{0}}\left(\tau^{-}\right)+\underline{f}^{-} \tau_{\underline{x}_{0}}\right)-\left(\underline{f}^{+}-\underline{f}^{-}\right) \tau_{\underline{x}_{0}} .
\end{aligned}
$$

Some further manipulation yields

$$
\underline{x}_{x_{0}}\left(\tau^{+}\right)=\underline{h}_{\underline{x}}^{*} \underline{x}_{x_{0}}\left(\tau^{-}\right)-\left(\underline{f}^{+}-\underline{h}_{\underline{x}}^{*} \underline{f}^{-}\right) \underline{\tau}_{\underline{x}_{0}}
$$

where $\underline{h}_{\underline{x}}^{*}$ and $\tau_{\underline{x}_{0}}$ are given by (55) and (48), respectively, as

$$
\begin{aligned}
\underline{h}_{\underline{x}}^{*} & =\left.\left(\underline{h_{x}}-\underline{h} \underline{h}_{y}\left(g_{y}^{-}\right)^{-1} g_{\underline{x}}^{-}\right)\right|_{\tau^{-}} \\
\tau_{\underline{x}_{0}} & =-\frac{\left.\left(s_{\underline{x}}-s_{y}\left(g_{\bar{y}}^{-}\right)^{-1} g_{\underline{x}}^{-}\right)\right|_{\tau^{-}} \underline{x}_{\underline{x}_{0}}\left(\tau^{-}\right)}{\left.\left(s_{\underline{x}}-s_{y}\left(g_{\bar{y}}^{-}\right)^{-1} g_{\underline{x}}^{-}\right)\right|_{\tau^{-}} \underline{f}^{-}}
\end{aligned}
$$


and $f^{+}, f^{-}$are given by (56). Differentiating and rearranging (52) gives

$$
y_{\underline{x}_{0}}\left(\tau^{+}\right)=-\left.\left(\left(g_{y}^{+}\right)^{-1} g_{\underline{x}}^{+}\right)\right|_{\tau^{+}} \underline{x}_{\underline{x}_{0}}\left(\tau^{+}\right) .
$$

Equations (57)-(60) together describe the trajectory sensitivity jump conditions.

In developing the jump conditions, we chose to use an arbitrary trigger function $s(\underline{x}, y)$. It can be seen that this function influences the jump conditions through $\tau_{\underline{x}_{0}}$. Reverting back to the original system description (13)-(16), the trigger function becomes

$$
s(\underline{x}, y)=y_{k}
$$

for some $k$. Therefore $s_{\underline{x}}=0$ and $s_{y}=\left[0 \cdots{ }^{k} \cdots 0\right]^{t}=\underline{1}_{k}$. Substituting into (59) gives

$$
\tau_{\underline{x}_{0}}=-\frac{\left.\underline{1}_{k}\left(\left(g_{y}^{-}\right)^{-1} g_{\underline{x}}^{-}\right)\right|_{\tau^{-}} \underline{x}_{\underline{x}_{0}}\left(\tau^{-}\right)}{\left.\underline{1}_{k}\left(\left(g_{\bar{y}}^{-}\right)^{-1} g_{\underline{x}}^{-}\right)\right|_{\tau^{-}} \underline{f}^{-}} .
$$

The compact form of the model enabled a neat development and representation of the jump conditions. However, as was the case at the end of Section IV, it is helpful to consider the jump conditions in terms of the full model description.

Consider first (58). It follows from the definition of $\underline{h}$ that

$$
\begin{aligned}
\underline{h}_{\underline{x}}^{*}= & \left(\left[\begin{array}{ccc}
I & 0 & 0 \\
h_{x} & h_{z} & h_{\lambda} \\
0 & 0 & I
\end{array}\right]\right. \\
& \left.-\left[\begin{array}{c}
0 \\
h_{y} \\
0
\end{array}\right]\left(g_{y}^{-}\right)^{-1}\left[\begin{array}{lll}
g_{x}^{-} & g_{z}^{-} & g_{\lambda}^{-}
\end{array}\right]\right)\left.\right|_{\tau^{-}} \\
= & {\left[\begin{array}{ccc}
I & 0 & 0 \\
h_{x}^{*} & h_{z}^{*} & h_{\lambda}^{*} \\
0 & 0 & I
\end{array}\right] }
\end{aligned}
$$

where

$$
h_{x}^{*}=\left.\left(h_{x}-h_{y}\left(g_{y}^{-}\right)^{-1} g_{x}^{-}\right)\right|_{\tau^{-}}
$$

and $h_{z}^{*}, h_{\lambda}^{*}$ follow the same pattern.

Now consider the expansion of (57). Recall that

$$
\underline{f}^{+}=\left[\begin{array}{c}
f^{+} \\
0 \\
0
\end{array}\right]
$$

and likewise for $\underline{f}^{-}$. Therefore

$$
\begin{aligned}
& {\left[\begin{array}{lll}
x_{x_{0}} & x_{z_{0}} & x_{\lambda} \\
z_{x_{0}} & z_{z_{0}} & z_{\lambda} \\
\lambda_{x_{0}} & \lambda_{z_{0}} & \lambda_{\lambda}
\end{array}\right]\left(\tau^{+}\right)} \\
& =\left[\begin{array}{ccc}
I & 0 & 0 \\
h_{x}^{*} & h_{z}^{*} & h_{\lambda}^{*} \\
0 & 0 & I
\end{array}\right]\left[\begin{array}{ccc}
x_{x_{0}} & x_{z_{0}} & x_{\lambda} \\
z_{x_{0}} & z_{z_{0}} & z_{\lambda} \\
\lambda_{x_{0}} & \lambda_{z_{0}} & \lambda_{\lambda}
\end{array}\right]\left(\tau^{-}\right) \\
& \quad-\left(\left[\begin{array}{c}
f^{+} \\
0 \\
0
\end{array}\right]-\left[\begin{array}{ccc}
I & 0 & 0 \\
h_{x}^{*} & h_{z}^{*} & h_{\lambda}^{*} \\
0 & 0 & I
\end{array}\right]\left[\begin{array}{c}
f^{-} \\
0 \\
0
\end{array}\right]\right) \tau_{\underline{x}_{0}}
\end{aligned}
$$

$$
=\left[\begin{array}{ccc}
x_{x_{0}} & x_{z_{0}} & x_{\lambda} \\
h_{x}^{*} \underline{x}_{x_{0}} & h_{x}^{*} \underline{x}_{z_{0}} & h_{x}^{*} \underline{x}_{\lambda} \\
\underline{\lambda}_{x_{0}} & \bar{\lambda}_{z_{0}} & \bar{\lambda}_{\lambda}
\end{array}\right]\left(\tau^{-}\right)-\left[\begin{array}{c}
f^{+}-f^{-} \\
-h_{x}^{*} f^{-} \\
0
\end{array}\right] \tau_{\underline{x}_{0}} .
$$

It can be seen from (63) that $\left[\lambda_{x_{0}} \lambda_{z_{0}} \lambda_{\lambda}\right]\left(\tau^{+}\right)=\left[\lambda_{x_{0}} \lambda_{z_{0}} \lambda_{\lambda}\right]$ $\left(\tau^{-}\right)$, i.e., that $\lambda_{\underline{x}_{0}}\left(\tau^{+}\right)=\lambda_{\underline{x}_{0}}\left(\tau^{-}\right)$. However, we established in Section IV that $\lambda_{x_{0}}$ remained constant over the intervals between events. Therefore, because $\lambda_{\underline{x}_{0}}\left(t_{0}\right)=\left[\begin{array}{lll}0 & 0 & I\end{array}\right]$, it follows that $\lambda_{\underline{x}_{0}}(t)=\left[\begin{array}{lll}0 & 0 & I\end{array}\right]$ for all time $t>t_{0}$.

The full model description leads to a useful simplification in the denominator of (61) [and likewise (59)]

$$
\begin{aligned}
\underline{1}_{k}\left(g_{\bar{y}}^{-}\right)^{-1} g_{\underline{x}}^{-} \underline{f}^{-} & =\underline{1}_{k}\left(g_{y}^{-}\right)^{-1}\left[\begin{array}{lll}
g_{x}^{-} & g_{z}^{-} & g_{\lambda}^{-}
\end{array}\right]\left[\begin{array}{c}
f^{-} \\
0 \\
0
\end{array}\right] \\
& =\underline{1}_{k}\left(g_{y}^{-}\right)^{-1} g_{x}^{-} f^{-} .
\end{aligned}
$$

The model (34)-(36) used to develop the jump conditions described a coincident switching and reset event. Consider the case where the event involves only the resetting of $z$, i.e., the algebraic equations do not switch from $g^{-}$to $g^{+}$, but are defined by a smooth function $g$. Then $g$ would be used for the (implicit) calculation of $y$ in both $f^{-}$and $f^{+}$in (56). Also, all occurrences of $g^{-}$in (58) and (59) [or alternatively (61)] would be replaced by $g$.

Now consider a switching event where $z$ is not reset, i.e., $\underline{x}^{+}=\underline{x}^{-}$. The reset function reverts to $h(x, y, z ; \lambda)=z$. It follows from (62) that $\underline{h}_{x}^{*}=I$ and the jump condition (57) becomes

$$
\underline{x}_{\underline{x}_{0}}\left(\tau^{+}\right)=\underline{x}_{\underline{x}_{0}}\left(\tau^{-}\right)-\left(\underline{f}^{+}-\underline{f}^{-}\right) \tau_{\underline{x}_{0}}
$$

which is consistent with [25]. Also, with no reset event, $\left(d \underline{x} / d \underline{x}_{0}\right)\left(\tau^{-}\right)=\left(d \underline{x} / d \underline{x}_{0}\right)\left(\tau^{+}\right)$and (64) follows directly from (49) and (51). Because of the structure of $\underline{f}^{+}$and $\underline{f}^{-}$, only $x_{\underline{x}_{0}}$ is updated at such an event, with $z_{\underline{x}_{0}}$ remaining constant through the event.

This latter case, with no state resetting, enables an intuitive interpretation of the jump conditions. Let $\Delta \underline{x}_{0}$ be a small perturbation in initial conditions. The corresponding change in the $\underline{x}$ component of the junction point will be $\Delta \underline{x} \approx\left(d \underline{x} / d \underline{x}_{0}\right) \Delta \underline{x}_{0}$ and in the junction time $\Delta \tau \approx \tau_{\underline{x}_{0}} \Delta \underline{x}_{0}$. It follows from (49) and (51) that

$$
\Delta \underline{x}=\underline{x}_{\underline{x}_{0}}^{-} \underline{x}_{0}+\underline{f}^{-} \Delta \tau=\underline{x}_{\underline{x}_{0}}^{+} \Delta \underline{x}_{0}+\underline{f}^{+} \Delta \tau
$$

where for convenience, the notation $\underline{x}_{x_{0}}^{-} \equiv \underline{x}_{x_{0}}\left(\tau^{-}\right)$and $\underline{x}_{x_{0}}^{+} \equiv$ $\underline{x}_{x_{0}}\left(\tau^{+}\right)$has been adopted. This relationship is shown graphically in Fig. 5.

The jump conditions occur as a consequence of the difference $\Delta \tau$ between the times when the nominal and perturbed trajectories reach the triggering hypersurface. Referring to Fig. 5 


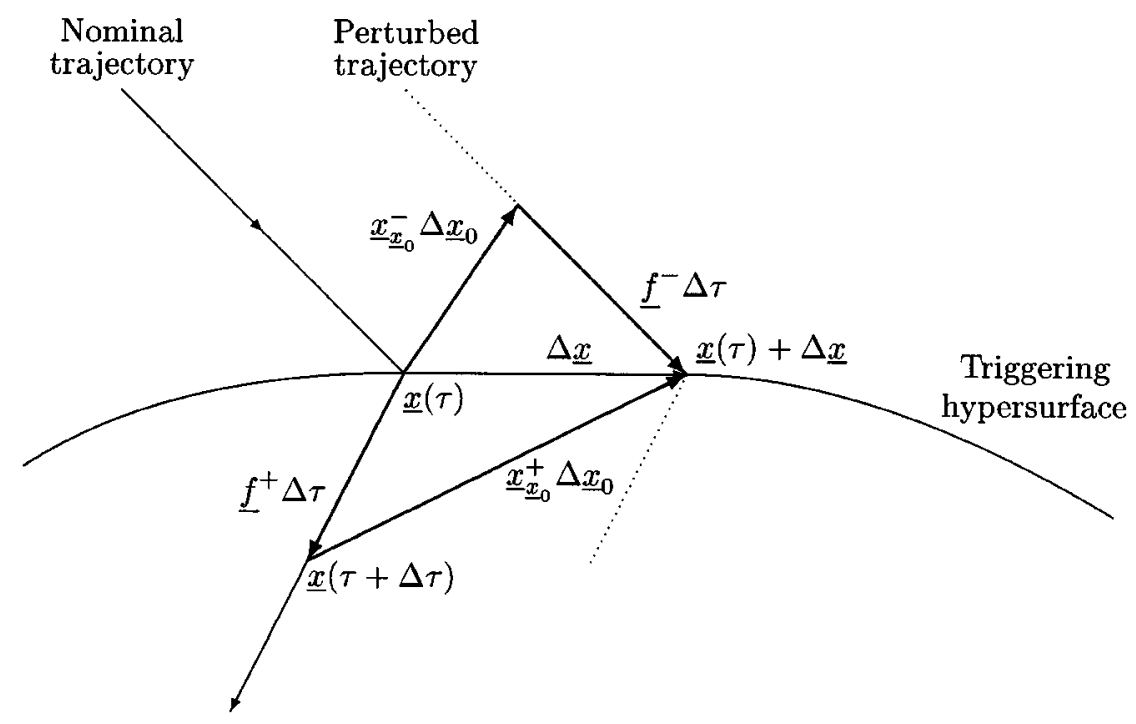

Fig. 5. Graphical view of jump conditions.

it can be seen that at time $\tau$, when the nominal trajectory encounters the hypersurface, the perturbed trajectory is at the point $\underline{x}(\tau)+\underline{x}_{\underline{x}_{0}}^{-} \underline{x}_{0}$. A further time $\Delta \tau$ is required for the perturbed trajectory to evolve from that point to $\underline{x}(\tau)+\Delta \underline{x}$ (a point on the hypersurface). However, during that time interval $\Delta \tau$, the nominal trajectory moves to the point $\underline{x}(\tau+\Delta \tau)=\underline{x}(\tau)+f^{+} \Delta \tau$. The perturbation $\underline{x}_{x_{0}}^{+} \Delta \underline{x}_{0}$ must therefore ensure that the perturbed trajectory is at the point $\underline{x}(\tau)+\Delta \underline{x}$ at time $\tau+\Delta \tau$, while taking into account the progression of the nominal trajectory. This is illustrated in the example of Section VI-A.

A few comments are in order.

- For clarity, Fig. 5 shows the perturbation $\underline{x}_{x_{0}}^{+} \Delta \underline{x}_{0}$ at time $\tau+\Delta \tau$. However the actual jump in sensitivity from $\underline{x}_{\underline{x}_{0}}^{-}$ to $\underline{x}_{\underline{x}_{0}}^{+}$occurs at the junction time $\tau$. There is no inconsistency, however, because $\Delta \tau$ is small so linearity can be assumed.

- The discussion has been based on positive $\Delta \tau$. However the sign of $\Delta \tau$ is unimportant.

- If the junction time is independent of changes in $\underline{x}_{0}$, i.e., $\tau_{x_{0}}=0$, or if $f$ is unchanged at switching, i.e., $f^{-}=$ $f^{+}$, then there will be no jump in the sensitivities, i.e., $\underline{x}_{\underline{x}_{0}}\left(\tau^{-}\right)=\underline{x}_{x_{0}}\left(\tau^{+}\right)$.

- Underlying the above discussion of jump conditions is the fact that the algebraic constraints $g$ are always satisfied. (There may be a switching of constraints at the triggering hypersurface, however.) The algebraic variables $y$ follow directly from solution of $g(\underline{x}, y)=0$ at all times.

- The more general jump conditions (57) do not allow such a clear interpretation of jump behavior. Similar statements can be made, though the influence of $\underline{h}_{\underline{x}}^{*}$ must be taken into account.

- It is unlikely that a trajectory would encounter two triggering hypersurfaces simultaneously. However the jump conditions extend naturally to such a nongeneric situation by introducing a small delay between the junction times and then taking the limit as that delay approaches zero.
Note that in setting up the fictitious delay, the ordering of the hypersurfaces must correspond to the order in which they would be encountered by the perturbed trajectory. This implies a knowledge of the perturbation $\Delta \underline{x}_{0}$.

\section{EXAMPLES}

\section{A. Example 1-Continued}

The phase portrait and time-domain response for this example were presented in Figs. 1 and 2. The sensitivity of that nominal trajectory to perturbation of $\lambda$, the slope of the steeper switching surface, is shown in Fig. 6. The two curves of that figure correspond to $\partial x_{1} / \partial \lambda$ and $\partial x_{2} / \partial \lambda$. It can be seen that there is zero sensitivity up to the point where the perturbed switching surface is first encountered. This is intuitively sensible, as the trajectory cannot be affected by the switching surface slope before it actually encounters the surface. At that first switching point and then at each subsequent switching, both sensitivities $\partial x_{1} / \partial \lambda$ and $\partial x_{2} / \partial \lambda$ undergo step changes. The influence of the perturbation diminishes as the equilibrium point is approached.

The analysis of Section III showed that trajectory sensitivities provide a first-order approximation of the change in a trajectory that results from parameter changes. The sensitivity $\partial x_{1} / \partial \lambda$ of Fig. 6 is used in Fig. 7 as an illustration. Fig. 7 shows the trajectory $x_{1}(t ; 2.75)$ corresponding to the nominal parameter value $\lambda=2.75$. (This is an enlargement of a portion of Fig. 2.) It also shows the trajectory $x_{1}(t ; 3.0)$ which corresponds to the perturbed parameter $\lambda=3.0$. The third curve

$$
x_{1}^{\text {approx }}(t ; 3.0)=x_{1}(t ; 2.75)+0.25 \frac{\partial x_{1}}{\partial \lambda}(t ; 2.75)
$$

uses the sensitivity $\left(\partial x_{1} / \partial \lambda\right)(t ; 2.75)$, evaluated with respect to the nominal trajectory, to approximate $x_{1}(t ; 3.0)$. The approximation $x_{1}^{\text {approx }}$ closely matches the perturbed trajectory $x_{1}(t ; 3.0)$ over each of the smooth segments of the trajectory, but diverges when the nominal trajectory $x_{1}(t ; 2.75)$ switches. 
The approximation and the perturbed trajectory again coincide when the perturbed trajectory switches.

The mismatch around the switching events is clarified by the discussion of jump conditions at the end of Section V-B. The perturbation of the slope parameter $\lambda$ from 2.75 to 3.0 results in a delay $\Delta \tau$ in the junction time $\tau$ for each event. The delays are apparent in Fig. 7, where the switching of $x_{1}(t ; 3.0)$ lags that of $x_{1}(t ; 2.75)$ at each event. The jump conditions at $\tau$ ensure that the sensitivities accurately reflect trajectory perturbations at and beyond the delayed junction time $\tau+\Delta \tau$. Over the intervening time interval $\Delta \tau$ the sensitivities cannot directly represent perturbations.

However, over the switching delay interval $\Delta \tau$, the perturbed trajectory can be accurately predicted through indirect use of the sensitivities. A procedure is given in Appendix A. Fig. 8 provides a comparison of the actual trajectory $x_{1}(t ; 3.0)$, the (direct) approximation from Fig. 7, and the approximation obtained using this refinement. The improvement in the approximation is clearly evident.

\section{B. Example 2-Continued}

Figs. 3 and 4 gave the phase portrait and time response of the nominal trajectory for this example. Fig. 9 illustrates the sensitivity of that trajectory to perturbation of the coefficient of restitution $\lambda$. The sensitivities of the algebraic state $y_{2}$ and the continuous state $x_{1}$ are shown. Due to the simple structure of this example, the sensitivity $\partial y_{2} / \partial \lambda$ remains constant between events. The continuous state $x_{1}$ is the integral of $y_{2}$, so the sensitivity $\partial x_{1} / \partial \lambda$ is the integral of the sensitivity $\partial y_{2} / \partial \lambda$. This can be seen in Fig. 9.

In Fig. 10, the trajectory sensitivity $\partial y_{2} / \partial \lambda$ is used to provide a first-order approximation of the trajectory of $y_{2}$ obtained when $\lambda$ is perturbed from 0.8 to 0.81 . The nominal and perturbed trajectories are shown as $y_{2}(t ; 0.8)$ and $y_{2}(t ; 0.81)$, respectively. The first order approximation is given by

$$
y_{2}^{\operatorname{approx}}(t ; 0.81)=y_{2}(t ; 0.8)+0.01 \frac{\partial y_{2}}{\partial \lambda}(t ; 0.8)
$$

where the sensitivity $\left(\partial y_{2} / \partial \lambda\right)(t ; 0.8)$ is evaluated for the nominal trajectory. The refinement of Appendix A has been used to improve the estimate over the switching delay intervals.

In this example, the oscillation period, i.e., the interval between corresponding reset events, decreases over time. In fact, it approaches zero. As a consequence, a small change in the coefficient of restitution $\lambda$ leads to the nominal and perturbed trajectories quickly moving out of phase. This can be seen in Fig. 10. Initially there is an excellent match between $y_{2}^{\text {approx }}$ and $y_{2}(t ; 0.81)$. However around $5.5 \mathrm{~s}$, the nominal and perturbed trajectories lose synchronism. The jump conditions still produce sensitivities which ensure $y_{2}^{\text {approx }}$ is close to $y_{2}(t ; 0.81)$ at time $\tau+\Delta \tau$. However, before that time is reached, the nominal trajectory encounters another event, and the sensitivities take another jump. Therefore, beyond $5.5 \mathrm{~s}, y_{2}^{\text {approx }}$ never catches up to $y_{2}(t ; 0.81)$ so the approximation is no longer valid.

This is an interesting case in that an equilibrium point is approached but can never be reached. Instead, the event triggering times accumulate at a finite time. Beyond that accumulation

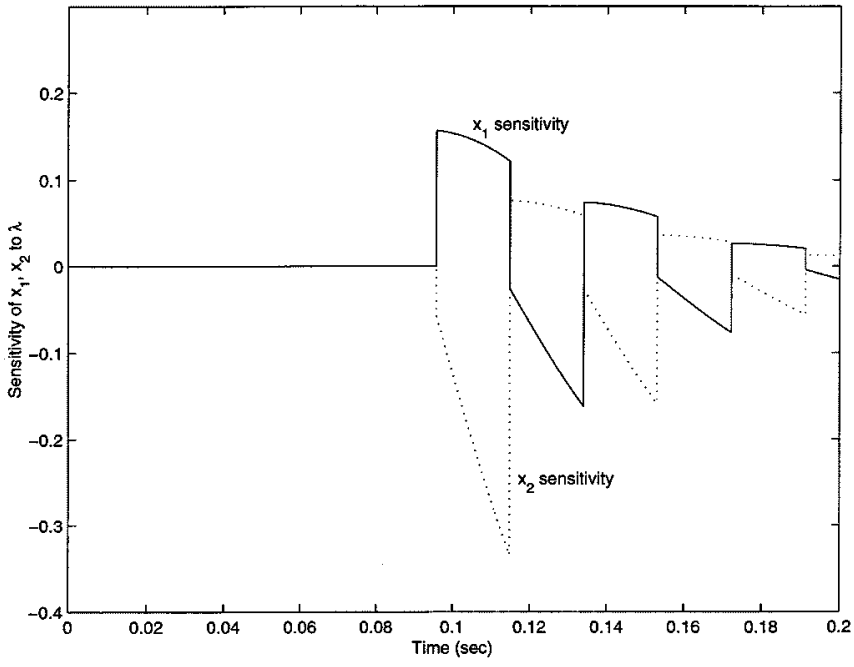

Fig. 6. Trajectory sensitivities for Example 1.

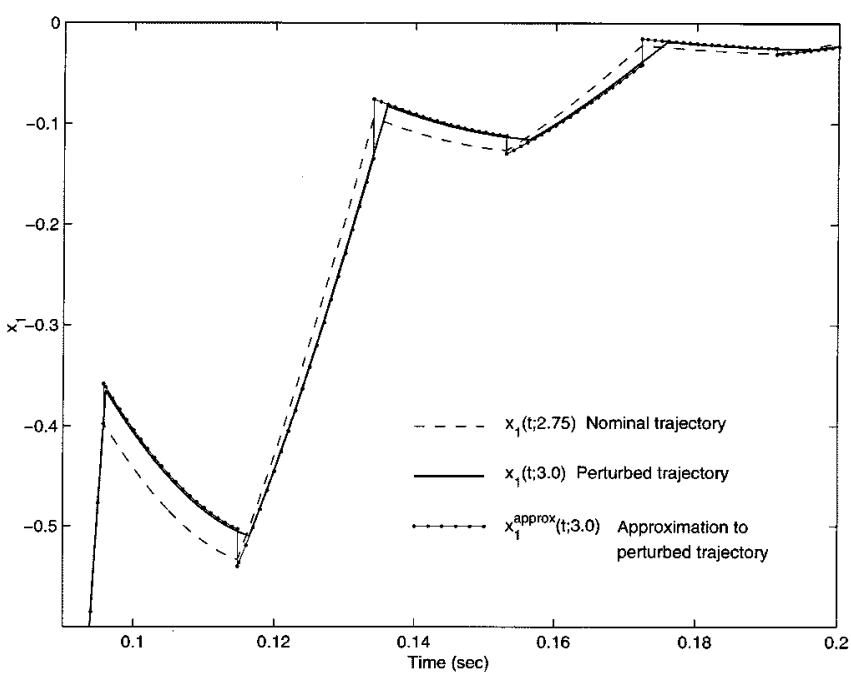

Fig. 7. Trajectory approximation for Example 1.

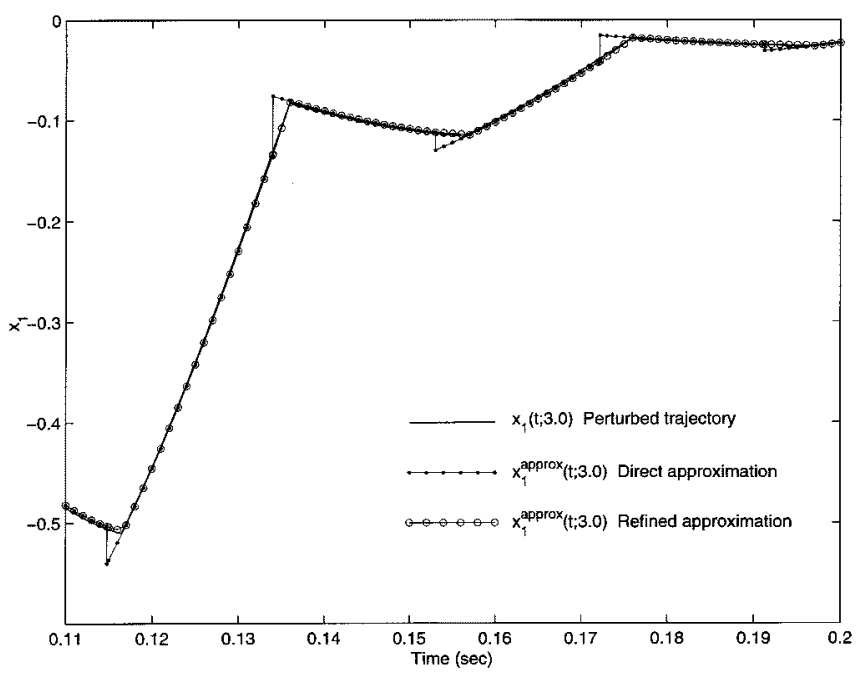

Fig. 8. Refined trajectory approximation for Example 1.

time, the solution is not defined in the usual sense. An alternative definition is required [26]. A consequence of this behavior 
is that a small increase in $\lambda$ results in the accumulation point being delayed to a time where the nominal trajectory did not even exist. The perturbed trajectory clearly cannot be approximated near the delayed accumulation point.

This example was repeated with the $\operatorname{sgn}\left(x_{2}\right)$ function of the original model replaced by a saturation function

$$
\operatorname{sat}\left(x_{2}\right)= \begin{cases}-1, & \text { for } x_{2}<-\frac{1}{K} \\ K x_{2}, & \text { for }\left|x_{2}\right| \leq \frac{1}{K} \\ 1, & \text { for } x_{2}>\frac{1}{K}\end{cases}
$$

This modified model approaches the original model as $K \rightarrow \infty$. However, for any finite $K$ an equilibrium point exists and the solution and trajectory sensitivities are well defined for all time. In this case, the approximation showed the same initial form as in Fig. 10. However, once $\left|x_{2}\right|<(1 / K)$, the error in the approximation diminished significantly.

\section{Example 3-Power System}

The small power system of Fig. 11 provides a more practical example of a system where continuous and discrete dynamics interact. The active power load has recovery dynamics [27], and is modeled by

$$
\begin{aligned}
\dot{x}_{p} & =\frac{1}{T_{p}}\left(P_{s}^{0}-P_{d}\right) \\
P_{d} & =x_{p}+P_{t} V_{3}^{2}
\end{aligned}
$$

where $x_{p}$ is the load state driving the actual load demand $P_{d}$. In response to a voltage step, the load undergoes an initial transient step change given by the term $P_{t} V_{3}^{2}$, followed by exponential recovery to the steady-state demand $P_{s}^{0}$. The rate of recovery is dictated by the load time constant $T_{p}$. In this example, the reactive power load is zero. Parameter values for the base case are given in Table I.

An important aim of this example is to illustrate the ability of the DAD structure (2)-(6) to model logic-based systems. Therefore, a relatively detailed representation of the automatic voltage regulator (AVR) of the tap changing transformer has been adopted. The logic flow of the AVR for low voltages, i.e., for increasing tap ratio, is outlined in Fig. 12. The full DAD model for this system is given in Appendix B. To assist in connecting AVR logic with the model, Fig. 12 shows the variables that are related to particular functions. It is clear that the model of Appendix B fits the DAD structure (2)-(6). Other power system control and protection devices have been modeled in a similar way in [28].

The system was disturbed at $t=10 \mathrm{~s}$ by increasing the impedance $X_{1}$ from $X_{1}^{\text {pre }}$ to $X_{1}^{\text {post }}$. This simulated the loss of a feeder from the supply point to the transformer. The behavior of the voltage at bus 3 is shown in Fig. 13, along with the load demand $P_{d}$. The system was clearly stable, although the voltage underwent a large excursion. The voltage stabilized to a value that was below the predisturbance level because the transformer encountered its maximum tap.

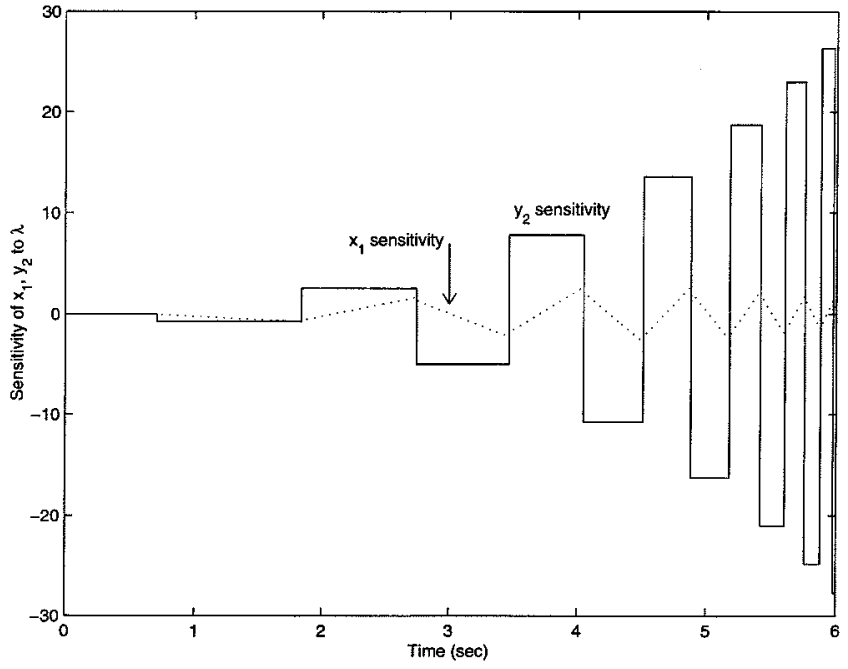

Fig. 9. Trajectory sensitivities for Example 2.

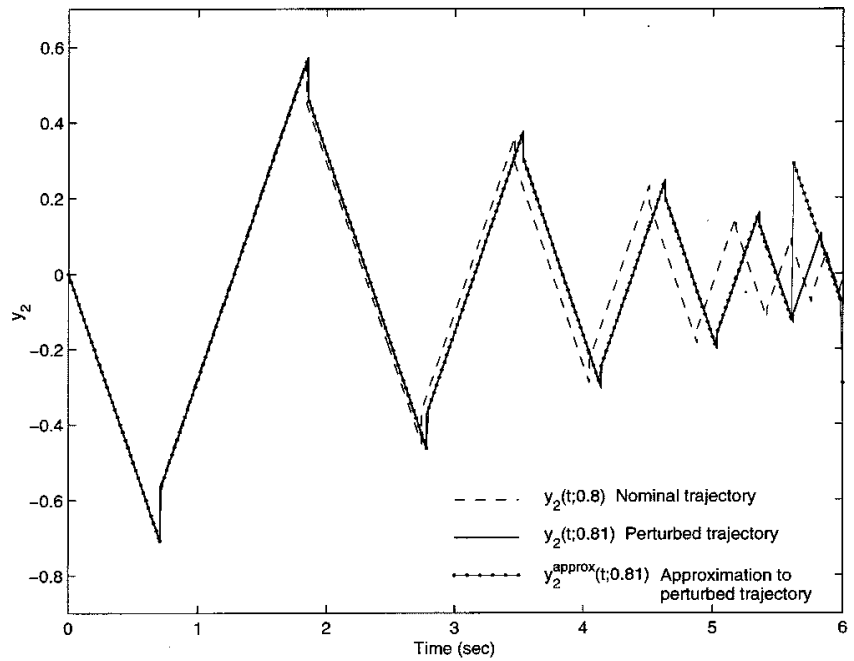

Fig. 10. Trajectory approximation for Example 2.

TABLE I

Base CASE PaRAmeter VALUES

\begin{tabular}{c|c|c|c|c|c}
\hline$T_{p}$ & $P_{s}^{0}$ & $P_{t}$ & $V_{\infty}$ & $X_{1}^{\text {pre }}$ & $X_{1}^{\text {post }}$ \\
\hline 5 & 0.4 & 0.4 & 1.05 & 0.25 & 0.65 \\
\hline \hline$X_{2}$ & $T_{\text {tap }}$ & $V_{\text {low }}$ & $n_{\text {step }}$ & $n_{\max }$ & $n_{\text {init }}$ \\
\hline 0.8 & 20 & 1.04 & 0.0125 & 1.1 & 1.0375 \\
\hline
\end{tabular}

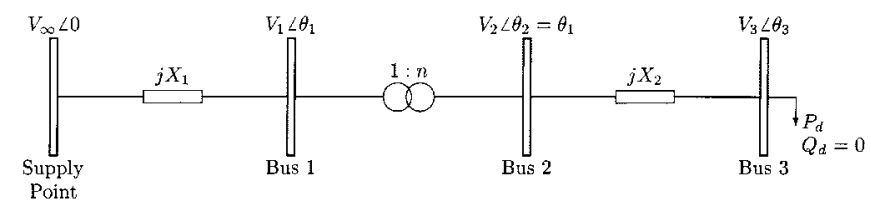

Fig. 11. Power system of Example 3.

Fig. 14 shows the sensitivity of the voltage $V_{3}$ trajectory to the parameters $T_{p}$ and $T_{\text {tap. }}$. These sensitivities are used in Fig. 15 to approximate voltage behavior for perturbation of both $T_{p}$ and $T_{\text {tap. }}$. Fig. 15 shows the trajectory $V_{3}(t ; 5,20)$ corresponding to the nominal parameter values $T_{p}=5, T_{\operatorname{tap}}=20$. It also shows 


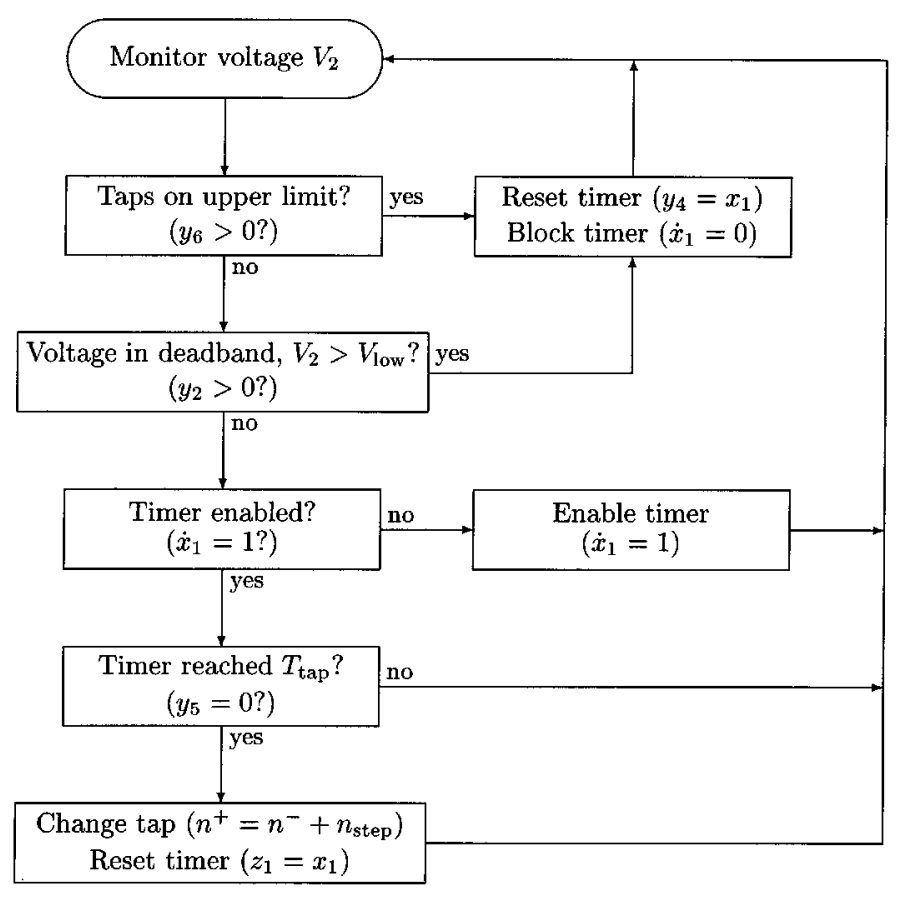

Fig. 12. Transformer AVR logic for increasing tap.

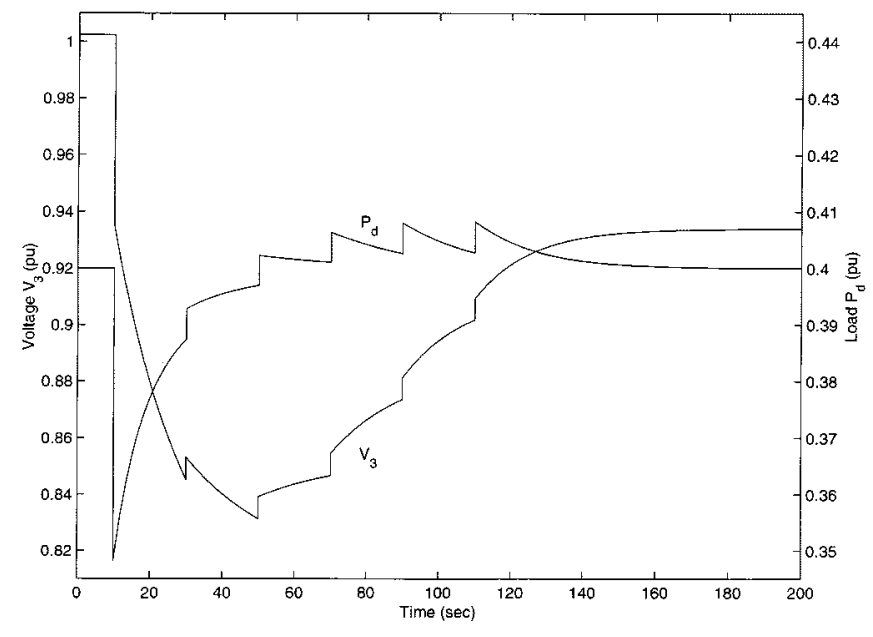

Fig. 13. Voltage and load behavior for Example 3.

the trajectory $V_{3}(t ; 5.5,22)$ which corresponds to perturbed parameters $T_{p}=5.5$ and $T_{\text {tap }}=22$. The third curve

$$
\begin{aligned}
& V_{3}^{\text {approx }}(t ; 5.5,22) \\
& \quad=V_{3}(t ; 5,20)+0.5 \frac{\partial V_{3}}{\partial T_{p}}(t ; 5,20)+2 \frac{\partial V_{3}}{\partial T_{\text {tap }}}(t ; 5,20)
\end{aligned}
$$

uses the sensitivities $\partial V_{3} / \partial T_{p}$ and $\partial V_{3} / \partial T_{\text {tap }}$, evaluated for the nominal case, to approximate $V_{3}(t ; 5.5,22)$. The procedure given in Appendix A has been used to estimate behavior over the $\Delta \tau$ periods, i.e., the periods between tapping of the nominal and perturbed systems. The approximation is clearly very good along the whole trajectory.

Trajectory sensitivities provide helpful insights in the analysis of system behavior. Consider first the sensitivity with respect to $T_{p}$. It can be seen from Fig. 14 that an increase in $T_{p}$

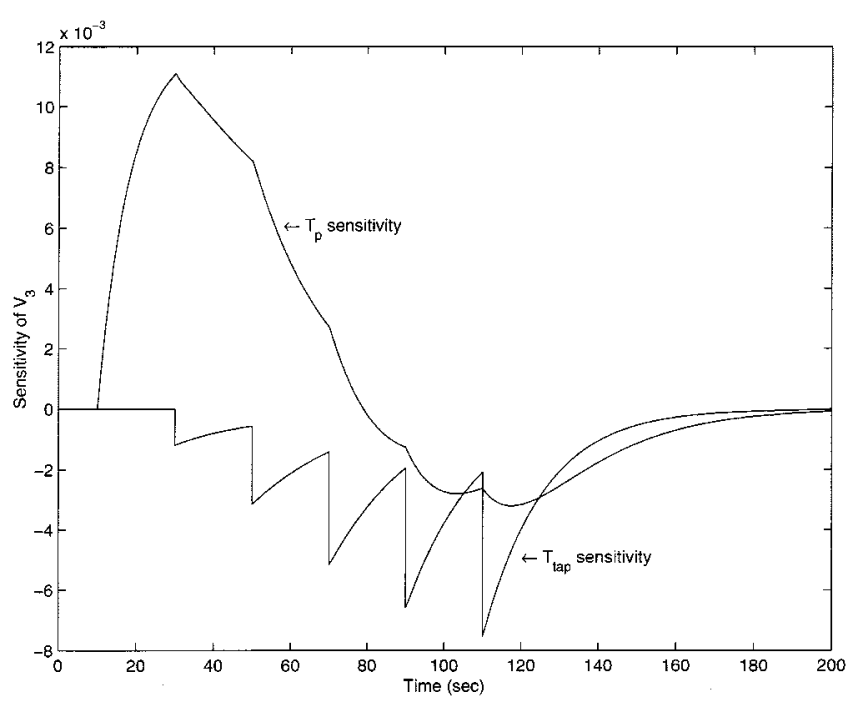

Fig. 14. Trajectory sensitivities for Example 3.

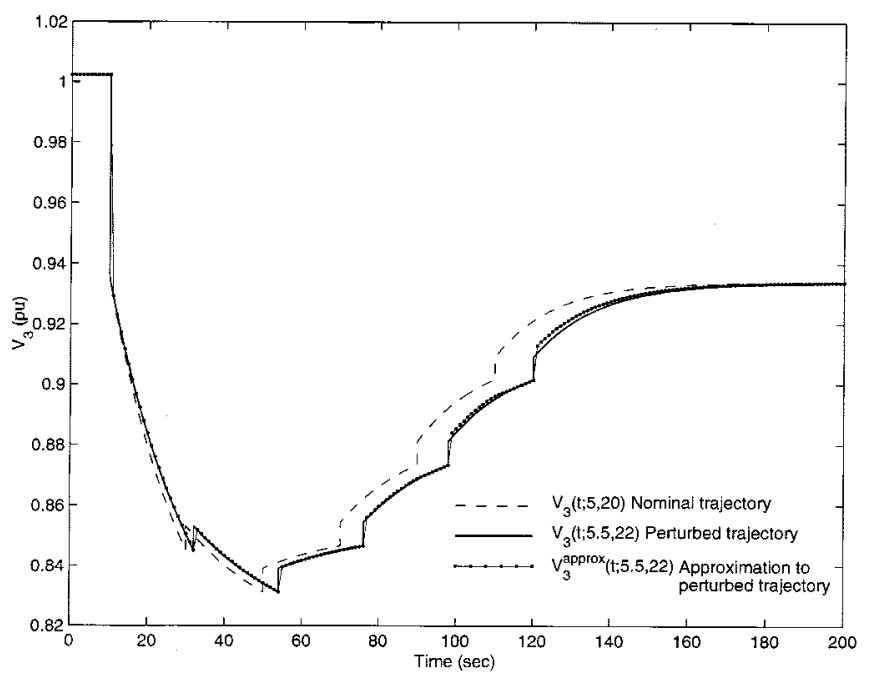

Fig. 15. Trajectory approximation for Example 3.

will lead to an increase in the voltage over the first $80 \mathrm{~s}$ of the trajectory, but after that it will result in a decrease in voltage. This is consistent with physical intuition. An increase in $T_{p}$ corresponds to slower load recovery. During the initial voltage drop, the load is less than its steady-state value $P_{s}^{0}$ (see Fig. 13). Therefore, slower load recovery means the load is smaller for longer, so the voltage is higher. However, over the latter section of the transient, whenever the voltage steps up due to a tap change the load overshoots $P_{s}^{0}$. So the slower recovery corresponds to the load staying higher for longer and, hence, to reduced voltage.

Now consider $T_{\text {tap }}$. From Fig. 14 it can be seen that an increase in $T_{\text {tap }}$ leads to a decrease in voltage. Again, this is consistent with intuition. It is clear that the voltage recovery is due to the increase in the tap ratio. Increasing $T_{\text {tap }}$ delays the tap changes, so the voltage stays lower for longer. The tap delay due to an increased $T_{\text {tap }}$ accumulates with each tap change. Therefore, the effect on the voltage becomes more pronounced with each subsequent tap change. This is evident in Fig. 14. 
For this simple example, the sensitivities do not provide qualitative information beyond that which is intuitively obvious. (Although they do provide quantitative information which is not otherwise available. For example, it can be seen from Fig. 14 that a $1 \mathrm{~s}$ change in $T_{p}$ would have a larger effect on the voltage trajectory than a $1 \mathrm{~s}$ change in $T_{\text {tap }}$.) However, for more complicated systems where the interpretation of parameter influences is not so straightforward sensitivities can be extremely useful. Such a situation is explored in [13].

It is shown in [15] and [25] that sensitivities can be used as an indicator of the proximity of a trajectory to the stability boundary. This is based on the fact that trajectories which are closer to the stability boundary will be more sensitive to parameters and initial conditions. This power system example nicely illustrates the result. Fig. 16 shows the $V_{3}$ trajectory for three values of the postdisturbance line impedance $X_{1}^{\text {post }}$, the base case value of 0.65 , along with slightly higher impedances 0.67 and 0.68. It is clear that higher values of line impedance result in a more stressed less secure system. However, the full significance of the increase in $X_{1}^{\text {post }}$ becomes much more apparent when the sensitivity of each trajectory to $T_{p}$, shown in Fig. 17, is viewed. When $X_{1}^{\text {post }}=0.68$ the system is extremely sensitive, indicating close proximity to instability. In fact, the system is unstable for $X_{1}^{\text {post }}=0.69$.

\section{NUMERICAL INTEGRATION}

\section{A. Introduction}

In Section IV, it was shown that the trajectory sensitivities evolve according to the linear time-varying DA system (27), (28). This system involves $(n+l+p) \times(n+l+p)$ DE's, along with $m \times(n+l+p)$ algebraic equations. [Recall, however, that $\lambda_{\underline{x}_{0}}$ is not interesting, so the number of DE's is effectively reduced to $(n+l) \times(n+l+p)$.] Therefore, for large systems such as power systems, the number of equations quickly becomes prohibitive. Of course, for many applications only a few of the sensitivities are required. For example, if the sensitivity of the trajectory to $i$ initial conditions and $j$ parameters was desired, then the number of extra DE's would be $(n+l) \times(i+j)$, along with $m \times(i+j)$ extra algebraic equations. Even so, the computational cost may be high.

Fortunately, by using an implicit numerical integration technique such as trapezoidal integration, the computational burden of obtaining the trajectory sensitivities can be reduced considerably. The details follow. Section VII-C then discusses the computation of junction points.

\section{B. Trapezoidal Numerical Integration}

The trapezoidal approach to numerical integration is quite standard and can be found in many references. In the power system context, [29] provides a clear presentation. The application to general DA systems is treated rigorously in [30]. A review of the basic ideas highlights a close link between the computation of the trajectory and of the associated sensitivities.

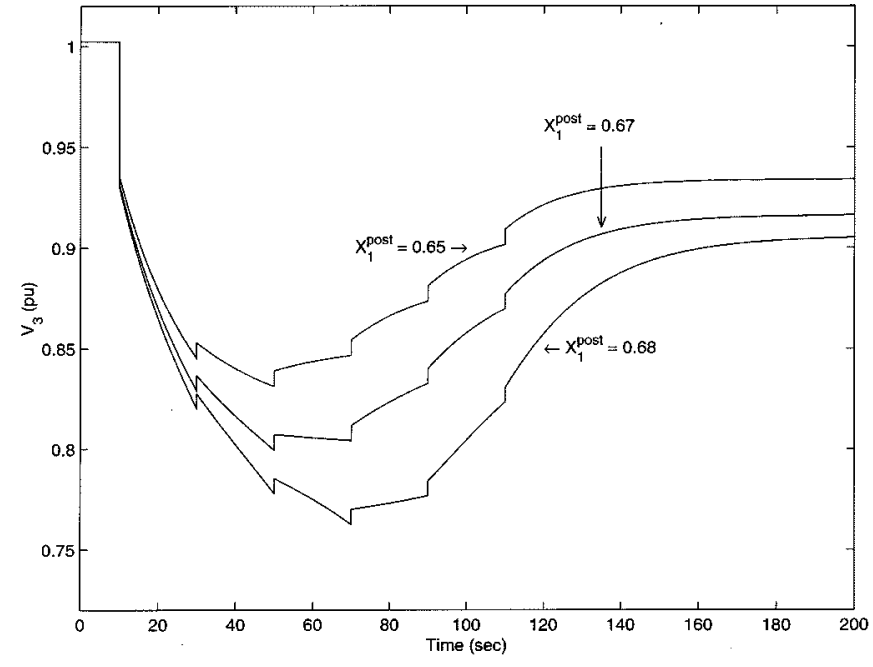

Fig. 16. Variation in voltage response with increasing $X_{1}^{\text {post }}$.

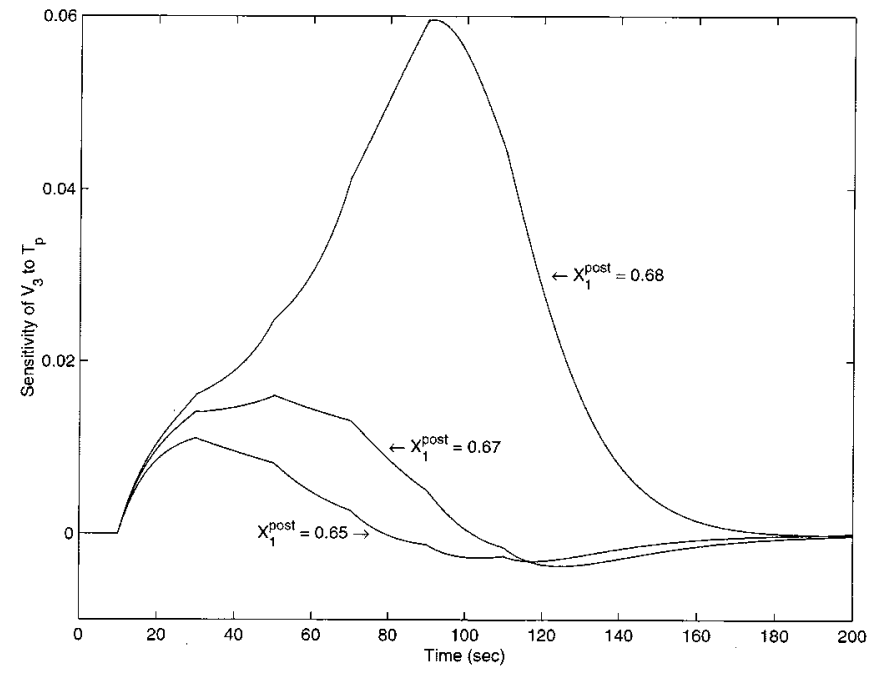

Fig. 17. Variation in trajectory sensitivity with increasing $X_{1}^{\text {post }}$.

Consider the DA system (18), (19) which describes behavior over the periods between events. We repeat the equations here for convenience

$$
\begin{aligned}
& \underline{\dot{x}}=\underline{f}(\underline{x}, y) \\
& 0=g(\underline{x}, y) .
\end{aligned}
$$

The trapezoidal approach approximates the DE's (67) by a set of algebraic difference equations coupled to the original algebraic equations (68), i.e.,

$$
\begin{aligned}
\underline{x}^{k+1} & =\underline{x}^{k}+\frac{\eta}{2}\left(\underline{f}\left(\underline{x}^{k}, y^{k}\right)+\underline{f}\left(\underline{x}^{k+1}, y^{k+1}\right)\right) \\
0 & =g\left(\underline{x}^{k+1}, y^{k+1}\right)
\end{aligned}
$$

where

superscript $k \quad$ indexes the time instant $t_{k}$; superscript $k+1$ indexes the time instant $t_{k+1}$; $\eta=t_{k+1}-t_{k} \quad$ integration time step.

Equations (69) and (70) describe the evolution of the states $\underline{x}, y$ from time instant $t_{k}$ to the next time instant $t_{k+1}$. Initial condi- 
tions for the time interval $\left(\tau_{j}, \tau_{j+1}\right)$ are given by $\underline{x}^{0}=\underline{x}\left(\tau_{j}^{+}\right)$ and $y^{0}=y\left(\tau_{j}^{+}\right)$.

Notice that (69) and (70) form a set of implicit nonlinear algebraic equations. Therefore, to solve for $\underline{x}^{k+1}, y^{k+1}$ given $\underline{x}^{k}$, $y^{k}$ requires the use of a nonlinear equation solver. The Newton iterative technique is commonly used. Rearranging (69) allows the algebraic equations to be written

$$
\begin{aligned}
F & \left(\underline{x}^{k+1}, y^{k+1}\right) \\
& =\left[\begin{array}{c}
\frac{\eta}{2} \underline{f}\left(\underline{x}^{k+1}, y^{k+1}\right)-\underline{x}^{k+1}+\frac{\eta}{2} \underline{f}\left(\underline{x}^{k}, y^{k}\right)+\underline{x}^{k} \\
g\left(\underline{x}^{k+1}, y^{k+1}\right)
\end{array}\right] \\
& =0 .
\end{aligned}
$$

Equation (71) has the form

$$
F(\varkappa)=0
$$

which is solved iteratively according to

$$
\varkappa_{i+1}=\varkappa_{i}-F_{\varkappa}\left(\varkappa_{i}\right)^{-1} F\left(\varkappa_{i}\right)
$$

where $F_{\varkappa}$ is the Jacobian of $F$ with respect to $\varkappa$, and has the structure

$$
F_{\varkappa}=\left[\begin{array}{ll}
\frac{\eta}{2} \underline{f}_{\underline{x}}-I & \frac{\eta}{2} \underline{f_{y}} \\
\underline{g}_{\underline{y}}
\end{array}\right] .
$$

Note that $i$ indexes the iterations of the equation solver, and is not related to the time index $k$. When (72) has converged, the solution $\varkappa$ provides $x^{k+1}$ and $y^{k+1}$.

Now consider the sensitivity equations (27) and (28). Using trapezoidal integration, they are approximated by

$$
\begin{aligned}
\underline{x}_{x_{0}}^{k+1} & =\underline{x}_{\underline{x}_{0}}^{k}+\frac{\eta}{2}\left(\underline{f}_{\underline{x}}^{k} \underline{x}_{\underline{x}_{0}}^{k}+\underline{f}_{y}^{k} y_{\underline{x}_{0}}^{k}+\underline{f}_{\underline{x}}^{k+1} \underline{x}_{\underline{x}_{0}}^{k+1}+\underline{f}_{y}^{k+1} y_{\underline{x}_{0}}^{k+1}\right) \\
0 & =g_{\underline{x}}^{k+1} \underline{x}_{\underline{x}_{0}}^{k+1}+g_{y}^{k+1} y_{\underline{x}_{0}}^{k+1} .
\end{aligned}
$$

Rearranging gives

$$
\begin{gathered}
{\left[\begin{array}{cc}
\frac{\eta}{2} \underline{f}_{\underline{x}}^{k+1}-I & \frac{\eta}{2} \underline{f}_{y}^{k+1} \\
g_{\underline{x}}^{k+1} & g_{y}^{k+1}
\end{array}\right]\left[\begin{array}{c}
\underline{x}_{\underline{x}_{0}}^{k+1} \\
y_{\underline{x}_{0}}^{k+1}
\end{array}\right]} \\
\quad=\left[\begin{array}{c}
-\frac{\eta}{2}\left(\underline{f_{x}^{k}} \underline{x}_{\underline{x}_{0}}^{k}+\underline{f}_{y}^{k} y_{\underline{x}_{0}}^{k}\right)-\underline{x}_{\underline{x}_{0}}^{k} \\
0
\end{array}\right]
\end{gathered}
$$

Therefore, $\underline{x}_{x_{0}}^{k+1}$ and $y_{\underline{x}_{0}}^{k+1}$ are obtained as the solution of a linear matrix equation. However, notice that the matrix to be factorized in solving (74) is exactly the Jacobian (73) used in solving for $\underline{x}^{k+1}$ and $y^{k+1}$ at (72). Furthermore, the solution of (72) requires the factorization of that Jacobian. Therefore, the factors of the left-hand matrix in (74) are available as a byproduct of calculating $\underline{x}^{k+1}$ and $y^{k+1}$. With those factors already available, the solution of (74) involves little extra computation.

\section{Computation of Junction Points}

Switching and reset events generically do not coincide with the time instants of the numerical integration process. However, for many applications is it important to find the exact time between integration time steps at which the event occurs. This is possible through a simple modification to the trapezoidal technique.

Referring to the compact DAD model (13)-(16), let $y_{i}=0$ trigger an event. Say $y_{i}<0$ at time instant $k$, but $y_{i}>0$ at instant $k+1$. Let $\eta^{*}$ be the (unknown) time from instant $k$ to the event. The variable $\eta^{*}$ can be found by solving (69) and (70) with $\eta$ free to vary, but with the extra constraint $y_{i}=0$. Because the extra variable is matched by an extra constraint, the Newton iterative technique can again be used to find the solution.

Having found the junction point, the appropriate switches in $g$ and/or update to $z$ should be made, then (70) resolved to obtain the postevent values of the algebraic variables $y$. The postevent values of $\underline{x}$ and $y$ provide the initial conditions for the next section of the trajectory. It can be convenient to use the time step $\eta-\eta^{*}$ for the first step after the event. This aligns subsequent points with the specified time step $\eta$.

\section{CONCLUSIONS}

Hybrid systems are characterized by the interaction between continuous and discrete-event dynamics. Power systems are an important example. The paper presents a DAD model which captures the rich variety of behavior that can be exhibited by such systems. Generally, because of the nonlinear nonsmooth behavior of hybrid systems, analysis is reliant on time-domain simulation. However, simulation can be cumbersome for anything beyond single scenarios. This paper develops trajectory sensitivities for hybrid systems, as a way of augmenting timedomain analysis.

Trajectory sensitivity analysis involves linearizing the system around a nominal trajectory, rather than an equilibrium point. It quantifies the changes in the system flow that result from perturbations in parameters and/or initial conditions. Calculating trajectory sensitivities over smooth sections of the flow is straightforward. An efficient numerical algorithm has been presented. However, discrete events generically induce jumps in the sensitivities. The paper establishes and explores those jump conditions.

In hybrid systems which exhibit accumulation points, the sensitivity information near an accumulation point may not be very useful. This is due to loss of event synchronism between the nominal and perturbed trajectories. However trajectory sensitivities still provide a good first-order approximation over the finite interval before synchronism is lost.

Trajectory sensitivities provide valuable insights into the influence of parameters on the dynamic behavior of systems. Properties which are not obvious from the actual system response are often evident in the sensitivities. This has been illustrated in the paper through a number of examples. Further, the gradient-type information given by trajectory sensitivities can be used in nonlinear least squares algorithms for applications such as parameter estimation [14], [31] and stability assessment [16]. This paper forms a basis for the extension of these and other applications to hybrid systems. 


\section{APPENDIX A \\ TRAJECTORY APPROXIMATION REFINEMENT}

This appendix suggests a procedure for refining the estimate of the perturbed trajectory over the period $\tau$ to $\tau+\Delta \tau$. For a given perturbation $\Delta \underline{x}_{0}$, the switching delay $\Delta \tau$ can be estimated from (59). Assume initially that $\Delta \tau>0$. Referring to Fig. 5, at time $\tau^{-}$the perturbed trajectory is at the point $\underline{x}^{\text {approx }}\left(\tau^{-}\right)=\underline{x}\left(\tau^{-}\right)+\underline{x}_{x_{0}}^{-} \Delta \underline{x}_{0}$. It follows that over the period $\tau$ to $\tau+\Delta \tau$, the perturbed trajectory is given by

$$
\begin{aligned}
& \left.\begin{array}{l}
\underline{x}^{\operatorname{approx}}(t)=\underline{x}\left(\tau^{-}\right)+\underline{x}_{\underline{x}_{0}}^{-} \Delta \underline{x}_{0}+\underline{f}^{-} \times(t-\tau) \\
y^{\operatorname{approx}}(t)=y\left(\tau^{-}\right)+y_{\underline{x}_{0}}^{-} \Delta \underline{x}_{0}-\left.\left(g_{y}^{-1} g_{\underline{x}}\right)\right|_{\tau^{-}} \underline{f}^{-} \times(t-\tau)
\end{array}\right\} \\
& \tau \leq t<\tau+\Delta \tau \text {. }
\end{aligned}
$$

At time $\tau+\Delta \tau$, the perturbed trajectory switches. In the general case where the event includes state resetting, we obtain

$$
\begin{aligned}
\underline{x}^{\operatorname{approx}}(\tau+\Delta \tau)^{+} & =\underline{h}\left(\underline{x}^{\text {approx }}(\tau+\Delta \tau), y^{\text {approx }}(\tau+\Delta \tau)\right) \\
& \approx \underline{x}(\tau)^{+}+\underline{h}_{\underline{x}}^{*} \underline{x}_{\underline{x}_{0}}^{-} \Delta \underline{x}_{0}+\underline{h}_{\underline{h}}^{*} \underline{f}^{-} \Delta \tau
\end{aligned}
$$

where $\underline{x}(\tau)^{+}$refers to the reset value. Using (57), we obtain

$$
\underline{x}^{\text {approx }}(\tau+\Delta \tau)^{+}=\underline{x}(\tau)^{+}+\underline{x}_{\underline{x}_{0}}^{+} \Delta \underline{x}_{0}+\underline{f}^{+} \Delta \tau .
$$

Alternatively, because $\underline{x}(\tau+\Delta \tau) \approx \underline{x}(\tau)^{+}+\underline{f}^{+} \Delta \tau$, (75) can be written

$$
\underline{x}^{\operatorname{approx}}(\tau+\Delta \tau)^{+}=\underline{x}(\tau+\Delta \tau)+\underline{x}_{\underline{x}_{0}}^{+} \underline{x}_{0} .
$$

For $\Delta \tau<0$ the perturbed trajectory switches at $\tau+\Delta \tau$, i.e., before the nominal trajectory. At $\tau+\Delta \tau$, just after switching, $\underline{x}^{\text {approx }}$ is again given by (75). Over the period $\tau+\Delta \tau$ to $\tau$, the perturbed trajectory in this case is given by

$$
\left.\begin{array}{l}
\underline{x}^{\text {approx }}(t)=\underline{x}(\tau)^{+}+\underline{x}_{\underline{x}_{0}}^{+} \Delta \underline{x}_{0}+\underline{f}^{+} \times(t-\tau) \\
y^{\text {approx }}(t)=y(\tau)^{+}+y_{\underline{x}_{0}}^{+} \Delta \underline{x}_{0}-\left.\left(g_{y}^{-1} g_{\underline{x}}\right)\right|_{\tau^{+}} \underline{f}^{+} \times(t-\tau) \\
\tau+\Delta \tau<t \leq \tau .
\end{array}\right\}
$$

At time $\tau$ we obtain

$$
\underline{x}^{\operatorname{approx}}(\tau)=\underline{x}(\tau)^{+}+\underline{x}_{\underline{x}_{0}}^{+} \Delta \underline{x}_{0}
$$

\section{APPENDIX B}

\section{MODEL FOR EXAMPLE 3}

The model for the system described by Figs. 11 and 12 is

$$
\begin{aligned}
\dot{x}_{1} & =y_{1} \\
\dot{x}_{p} & =\left(P_{s}^{0}-P_{d}\right) / T_{p} \\
0 & =P_{d}-x_{p}-P_{t} V_{3}^{2} \\
0 & =y_{3}-y_{4}+z_{1} \\
0 & =y_{6}-n+n_{\max }-n_{\text {step }} / 2 \\
0 & =n V_{1}-V_{2}
\end{aligned}
$$

$$
\begin{aligned}
& 0=y_{1}-1 \\
& \left.\begin{array}{l}
0=y_{1} \\
0=y_{1}-x_{1}
\end{array}\right\} \\
& y_{2}<0 \\
& \left.0=y_{4}-x_{1}\right\} \\
& y_{2}>0 \\
& 0=y_{2}-V_{2}+V_{\mathrm{low}} \\
& y_{6}<0 \\
& 0=y_{2}-1 \\
& y_{6}>0 \\
& 0=y_{5}-x_{1}+z_{1}+T_{\text {tap }} \\
& y_{3}<0 \\
& 0=y_{5}-x_{1}+y_{4}+T_{\text {tap }} \\
& \left.\begin{array}{l}
z_{1}^{+}=x_{1}^{-} \\
n^{+}=n^{-}+n_{\text {step }}
\end{array}\right\} \\
& y_{3}>0 \\
& \text { when } y_{5}=0
\end{aligned}
$$

plus real and reactive power balance equations for buses 1 and 3.

\section{ACKNOWLEDGMENT}

The authors would like to thank the reviewers for their valuable comments.

\section{REFERENCES}

[1] S. Pettersson, "Analysis and design of hybrid systems," Ph.D. thesis, Dept. Signals and Systems, Chalmers Univ. Technol., Göteborg, Sweden, 1999.

[2] I. A. Hiskens, "Analysis tools for power systems - Contending with nonlinearities," Proc. IEEE, vol. 83, pp. 1573-1587, Nov. 1995.

[3] M. A. Pai, Energy Function Analysis for Power System Stability. Boston, MA: Kluwer, 1989.

[4] H. Ye, A. N. Michel, and L. Hou, "Stability theory for hybrid dynamical systems," in Proc. 34th Conf. Decision Control, New Orleans, LA, Dec. 1995, pp. 2679-2684.

[5] P. M. Frank, Introduction to System Sensitivity Theory. New York, NY: Academic, 1978.

[6] P. V. Kokotović and R. S. Rutman, "Sensitivity of automatic control systems (survey)," Automation Remote Contr., vol. 26, pp. 727-749, 1965.

[7] R. Tomović and M. Vukobratović, General Sensitivity Theory. New York, NY: Elsevier, 1972.

[8] H. Khalil, Nonlinear Systems, 2nd ed. Englewood Cliffs, NJ: PrenticeHall, 1996.

[9] J. B. Cruz Jr., System Sensitivity Analysis. Stroudsburg, PA: Dowden, Hutchinson and Ross, 1973.

[10] Y.-C. Ho, "Performance evaluation and perturbation analysis of discrete event dynamic systems," IEEE Trans. Automat. Contr., vol. 32, pp. 563-572, July 1987.

[11] Y.-C. Ho and X.-R. Cao, Perturbation Analysis of Discrete Event Dynamic Systems. Norwell, MA: Kluwer, 1991.

[12] X.-R. Cao, "A comparison of the dynamics of continuous and discrete event systems," Proc. IEEE, vol. 77, pp. 7-13, Jan. 1989.

[13] I. A. Hiskens and M. Akke, "Analysis of the Nordel power grid disturbance of January 1, 1997 using trajectory sensitivities," IEEE Trans. Power Syst., vol. 14, pp. 987-994, Aug. 1999.

[14] S. M. Benchluch and J. H. Chow, "A trajectory sensitivity method for the identification of nonlinear excitation system models," IEEE Trans. Energy Conversion, vol. 8, pp. 159-164, June 1993.

[15] M. J. Laufenberg and M. A. Pai, "A new approach to dynamic security assessment using trajectory sensitivities," IEEE Trans. Power Syst., vol. 13, pp. 953-958, Aug. 1998.

[16] I. A. Hiskens, M. A. Pai, and P. W. Sauer, "An iterative approach to calculating dynamic ATC," in Proc. Int. Symp. Bulk Power System Dynamics Control-IV, Santorini, Greece, Aug. 1998.

[17] M. S. Branicky, V. S. Borkar, and S. K. Mitter, "A unified framework for hybrid control: Model and optimal control theory," IEEE Trans. Automat. Contr., vol. 43, pp. 31-45, Jan. 1998.

[18] A. Deshpande and P. Varaiya, "Viable control of hybrid systems," in Proc. 35th Conf. Decision Control, Kobe, Japan, Dec. 1996, pp. 1196-1201.

[19] J. H. Taylor, "Rigorous handling of state events in Matlab," in Proc. 4th IEEE Conf. Control Applications, Albany, NY, Sept. 1995, pp. 156-161.

[20] W. Fleming, Functions of Several Variables. New York: Springer-Verlag, 1977.

[21] J. H. Taylor and D. Kebede, "Modeling and simulation of hybrid systems," in Proc. 34th Conf. Decision Control, New Orleans, LA, Dec. 1995, pp. 2685-2687. 
[22] V. Venkatasubramanian, X. Jiang, H. Schättler, and J. Zaborszky, "Current status of the taxonomy theory of large power system dynamics-DAE systems with hard limits," in Proc. NSF/ECC Workshop on Bulk Power System Voltage Phenomena III, Davos, Switzerland, Aug. 1994, pp. 15-103.

[23] I. A. Hiskens and D. J. Hill, "Energy functions, transient stability and voltage behavior in power systems with nonlinear loads," IEEE Trans. Power Syst., vol. 4, no. 4, pp. 1525-1533, Nov. 1989.

[24] S. S. Sastry and C. A. Desoer, "Jump behaviour of circuits and systems," IEEE Trans. Circuits Syst., vol. 28, no. 12, pp. 1109-1124, Dec. 1981.

[25] I. A. Hiskens, "Trajectory sensitivity analysis of discontinuous differential-algebraic systems," Department of Electrical and Computer Engineering, The University of Newcastle, Tech. Rep. EE9717, May 1997.

[26] A. F. Filippov, Differential Equations with Discontinuous Righthand Sides. Amsterdam, The Netherlands: Kluwer, 1988

[27] D. J. Hill, "Nonlinear dynamic load models with recovery for voltage stability studies," IEEE Trans. Power Syst., vol. 8, pp. 166-176, Feb. 1993.

[28] L. G. Perez, A. J. Flechsig, and V. Venkatasubramanian, "Modeling the protective system for power system dynamic analysis," IEEE Trans. Power Syst., vol. 9, pp. 1963-1973, Nov. 1994.

[29] P. W. Sauer and M. A. Pai, Power System Dynamics and Stability. Englewood Cliffs, NJ: Prentice-Hall, 1998.

[30] K. E. Brenan, S. L. Campbell, and L. Petzold, Numerical Solution of Initial-Value Problems in Differential-Algebraic Equations. Philadelphia, PA: SIAM, 1995.

[31] I. A. Hiskens and A. J. A. Koeman, "Power system parameter estimation," J. Electr. Electron. Eng. Aust., vol. 19, no. 1, pp. 1-8, June 1999.

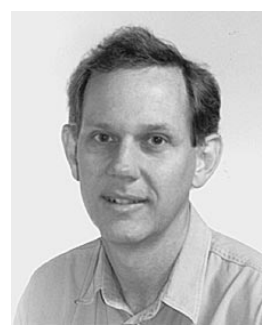

Ian A. Hiskens (S'78-M'84-SM'96) received the B.Eng. and B.App.Sc. degrees from the Capricornia Institute of Advanced Education, Rockhampton, Australia, in 1980 and 1983, respectively, and the $\mathrm{Ph} . \mathrm{D}$. degree from the University of Newcastle, Newcastle, Australia, in 1990.

From 1980 to 1992, he was with the Queensland Electricity Supply Industry. He was a Senior Lecturer in the Department of Electrical and Computer Engineering at the University of Newcastle from 1992 to 1999 and is currently a Visiting Associate Professor at the University of Illinois at Urbana-Champaign.

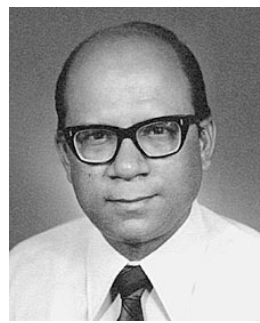

M. A. Pai (F'86) received the B.E. degree from the University of Madras, Madras, India, in 1953 and the M.S. and Ph.D. degrees from the University of California, Berkeley, in 1958 and 1961, respectively.

$\mathrm{He}$ was on the faculty of the Indian Institute of Technology, Kanpur from 1963 to 1981. Since 1981 he has been on the faculty of the University of Illinois at Urbana-Champaign as a Professor of electrical and computer engineering. 